\title{
Clinical Characteristics, Treatment Modalities, and Potential Contributing and Prognostic Factors in Patients with Bone Metastases from Gynecological Cancers: A Systematic Review
}

\author{
Francesca Salamanna ${ }^{1}\left(\mathbb{D}\right.$, Anna Myriam Perrone ${ }^{2,3,4}{ }^{\circledR}$, Deyanira Contartese ${ }^{1, *}$, Veronica Borsari ${ }^{1}$, \\ Alessandro Gasbarrini ${ }^{5}$, Silvia Terzi ${ }^{5}$, Pierandrea De Iaco ${ }^{2,3,4}$ and Milena Fini ${ }^{1}$ \\ 1 Complex Structure Surgical Sciences and Technologies, IRCCS Istituto Ortopedico Rizzoli, \\ 40136 Bologna, Italy; francesca.salamanna@ior.it (F.S.); veronica.borsari@ior.it (V.B.); milena.fini@ior.it (M.F.) \\ 2 Division of Oncologic Gynecology, IRCCS Azienda Ospedaliero-Universitaria di Bologna, \\ 40138 Bologna, Italy; myriam.perrone@aosp.bo.it (A.M.P.); pierandrea.deiaco@unibo.it (P.D.I.) \\ 3 Department of Medical and Surgical Sciences (DIMEC), University of Bologna, 40126 Bologna, Italy \\ 4 Centro di Studio e Ricerca delle Neoplasie Ginecologiche (CSR), University of Bologna, 40138 Bologna, Italy \\ 5 Department of Oncologic and Degenerative Spine Surgery, IRCCS Istituto Ortopedico Rizzoli, \\ 40136 Bologna, Italy; alessandro.gasbarrini@ior.it (A.G.); silvia.terzi@ior.it (S.T.) \\ * Correspondence: deyanira.contartese@ior.it
}

Citation: Salamanna, F.; Perrone, A.M.; Contartese, D.; Borsari, V.; Gasbarrini, A.; Terzi, S.; De Iaco, P.; Fini, M. Clinical Characteristics, Treatment Modalities, and Potential Contributing and Prognostic Factors in Patients with Bone Metastases from Gynecological Cancers: A Systematic Review. Diagnostics 2021, 11, 1626. https://doi.org/10.3390/ diagnostics11091626

Academic Editor: Giorgio Treglia

Received: 23 August 2021

Accepted: 30 August 2021

Published: 6 September 2021

Publisher's Note: MDPI stays neutral with regard to jurisdictional claims in published maps and institutional affiliations.

Copyright: (c) 2021 by the authors. Licensee MDPI, Basel, Switzerland. This article is an open access article distributed under the terms and conditions of the Creative Commons Attribution (CC BY) license (https:// creativecommons.org/licenses/by/ $4.0 /)$.

\begin{abstract}
The purpose of this study is to review the clinical characteristics, treatment modalities, and potential contributing and prognostic factors of bone metastases from gynecological cancers (GCs). A systematic literature search on PubMed, Scopus, Web of Science Core Collection and Cochrane Central Register of Controlled Trials databases was conducted. Thirty-one studies, all retrospective, were included in this review, for a total of 2880 patients with GC bone metastases. Primary tumors leading to bone metastases included endometrial cancer (EC), cervical cancer (CC), ovarian cancer $(\mathrm{OC})$, uterine sarcoma (US) and vulvar cancer $(\mathrm{VuC})$, mainly with an International Federation of Gynecology and Obstetrics (FIGO) Stage of III and IV. The main bone metastatic lesion site was the vertebral column, followed by the pelvic bone and lower extremity bones. The median survival rate after bone metastases diagnosis ranged from 3.0 to 45 months. The most frequent treatments were palliative and included radiotherapy and chemotherapy, followed by surgery. The findings of this review give a first dataset for a greater understanding of GC bone metastases that could help clinicians move toward a more "personalized" and thus more effective patient management.
\end{abstract}

Keywords: bone metastases; gynecological cancer; clinical studies; systematic review

\section{Introduction}

Bone is a common site of metastases and frequently indicates a short-term prognosis in patients with cancer. The acute effects of bone metastases include skeletal-related events (SREs; characterized by pathological fracture, the need for radiotherapy or bone surgery, spinal cord compression, and hypercalcemia), which may compromise patients physiological function and quality of life. Once cancer spreads to the bones, it can seldom be cured, but frequently it can be treated to reduce its growth. Breast, prostate, lung, kidney, and thyroid cancers are most likely to spread to the bone, and numerous studies in have analyzed their clinical characteristics, treatment, and potential contributing and prognostic factors [1]. However, other cancer types can also metastasize to bone; the increase in overall survival in patients with primary and/or metastatic cancer increases the probability that patients can develop bone metastases during their disease. This is also true for gynecological cancers (GCs), which include cervical, ovarian, uterine, vaginal, and vulvar malignancies. Worldwide, GCs affect millions of women across all ages [2]. It is estimated that in the United States, the number of women with GCs is approximately 80,000 /year [3]. However, differences in the incidence of GCs in diverse areas of the world 
have been detected. In the European Union, the estimated number of newly diagnosed cases is higher, with about 200,000 women/year [4]. Despite the enormous progress and advancements in the preventive, diagnostic, and therapeutic interventions for GCs, distant metastases and recurrence are still the leading cause of death in patients. Metastases from GCs differ depending on the cancer type. Cervical cancer (CC), endometrial cancer (EC), and uterine sarcomas (US) mainly spread to the lung and liver, while ovarian cancer (OC) primarily metastasizes locally within the peritoneum and pelvis [5-7]. However, distant sites such as bone may be also involved [8-12]. Bone metastases from GCs were detected in about $1.1-5.2 \%$ of patients with CC [8], in $1.2 \%$ of patients with OC [ 9$]$, in $2-8 \%$ of patients with EC [10], and rarely in vulvar ( $\mathrm{VuC})[11]$ and vaginal cancers $(\mathrm{VaC})$ [12]. Despite the uncommon incidence of GC bone metastases, the prolonged local disease control and the easy availability of advanced imaging techniques are increasing this incidence, and more and more anecdotal evidence is reported in the literature $[8,13,14]$. Not unexpectedly, these complications seriously affect quality of life, performance status, independent functioning, and survival. Conventionally, radiotherapy is considered the first line treatment in the management of GC metastatic bone lesions; however, impending or existing pathological fractures, spinal cord compression, unbearable pain, and resistance to radiotherapy are indications/complications that in most cases require a surgical approach [15]. Obviously, considering GC heterogeneity, i.e., entity, type, stage, grade, as well as patient heterogeneity (i.e., physical status, age, life styles, menopausal status), the "optimal" management can change [15]. Consequently, comparability between patients with GC bone metastasis in clinical studies is still limited and complex due to specific differences between tumors of the same type in different patients and between cancer cells within a tumor. This complexity is even more accentuated since few single-center, multi-center, and population-based studies report specific details on patients with GC bone metastasis. Furthermore, a systematic review of data on different types of GC bone metastasis, also considering all the anecdotal evidence present in literature, is, to date, lacking. Thus, a general overview of indications on the evaluation, incidence, complications, prognostic characteristics, and management of GCs that metastasize to bone remains a challenge for both gynecologic oncologists and researchers. Here, we conducted a systematic review, focusing on clinical characteristics, treatment, and potential contributing and prognostic factors of bone metastases in GCaffected patients in order to have a first dataset able to give a greater understanding of this pathological condition and to guide clinicians towards a more "personalized" patient management.

\section{Results}

\subsection{Study Selection and Characteristics}

The initial literature search retrieved 4102 studies. Of those, 645 studies were identified using PubMed, 2469 using Scopus, 939 in the Web of Science Core Collection, and 49 using the Cochrane Central Register of Controlled Trials. After screening the title and abstract, 262 articles were submitted to a public reference manager to eliminate duplicate articles. The resulting 79 complete articles were then reviewed to establish whether the publications met the inclusion criteria, and 31 studies were considered eligible for this review. All the included studies were retrospective. Search strategy and study inclusion and exclusion criteria are detailed in Figure 1. 

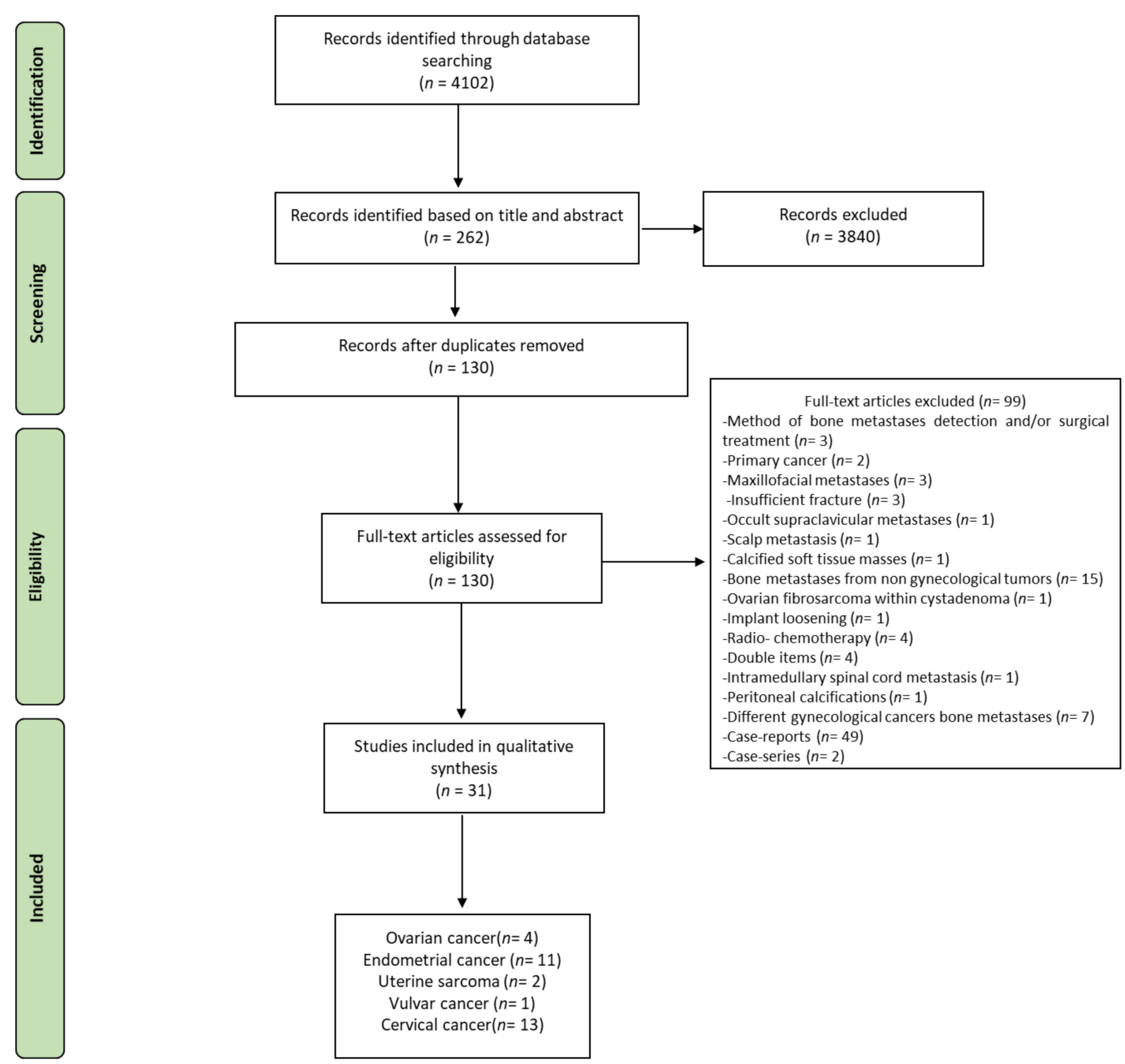

Figure 1. Preferred Reporting Items for Systematic Reviews and Meta-Analyses (PRISMA) flowchart for the selection of studies.

\subsection{Assessment of Methodological Quality}

The risks of bias assessments for each study are summarized in Table 1. Of the 31 articles eligible for the review, we found that all studies were retrospective. Using the NIH Quality Assessment Tool for Observational Cohort Studies [16,17], we rated one retrospective study at a "good" quality rating and 30 studies at a "fair" quality rating (Table 1). For the 30 retrospective studies at a "fair" quality rating, the principal missing quality assessment criteria were sample size justification and blinded assessors to the exposure of participants (Table 1). However, 14 studies also showed bias for the description of the study population and for the presence of potential confounding variables not measured and/or statistically adjusted (Table 1).

\subsection{Characteristics of the Studies}

Characteristics of the studies regarding geographical region, execution date, type, patient number, bone metastases occurrence, median/mean age, menopausal status, primary tumor histopathology and clinical stage and grade, survival, and/or follow-up period after bone metastases are described in Table 2. The 31 studies included in this review, selected 
according to the inclusion criteria, were all published between 2013 and 2021, but the time horizon covered for patient selection ranged between 1984 and 2019. All the studies were retrospective; 4 were on OC, 11 on EC, 2 on US, 1 on VuC, and 13 on CC. No studies on $\mathrm{VaC}$ bone metastases were founded with our search strategy.

Table 1. Quality assessment tool for retrospective cohort studies.

\begin{tabular}{|c|c|c|c|c|c|c|c|c|c|c|c|c|c|c|}
\hline \multirow{2}{*}{ Reference } & \multicolumn{14}{|c|}{ Criteria } \\
\hline & 1 & 2 & 3 & 4 & 5 & 6 & 7 & 8 & 9 & 10 & 11 & 12 & 13 & 14 \\
\hline \multicolumn{15}{|c|}{ Ovarian cancer } \\
\hline Deng et al. 2018 [18] & & & & & & & $\overline{\mathrm{NA}}$ & NA & & NA & & & & \\
\hline Sehouli et al. 2013 [19] & & & & & & & NA & NA & & NA & & & & \\
\hline Zhang et al. 2013 [20] & & & & & $\mathrm{CD}$ & & NA & NA & & NA & & & & \\
\hline Zhang et al. 2019 [21] & & & & & & & NA & NA & & NA & & & & \\
\hline \multicolumn{15}{|c|}{ Endometrial cancer } \\
\hline Guo et al. 2020 [22] & & & & & & & NA & NA & & NA & & & & \\
\hline Hong et al. 2021 [23] & & & & & & & NA & NA & & NA & & & & \\
\hline Kimyon et al. 2016 [24] & & & & & & & NA & NA & & NA & & & & \\
\hline Li et al. 2020 [25] & & & & & & & NA & NA & & NA & & & & \\
\hline Liu et al. 2020 [26] & & & & & & & NA & NA & & NA & & & & \\
\hline Mao et al. 2020 [27] & & & & & & & NA & NA & & NA & & & & \\
\hline McEachron et al. 2020 [28] & & & & & & & NA & NA & & NA & & & & \\
\hline Ouldamer et al. 2019 [29] & & & & & & & NA & NA & & NA & & & & \\
\hline Takeshita et al. 2016 [30] & & & & & & & NA & NA & & NA & & & & \\
\hline Uccella et al. 2013 [31] & & & & & & & NA & NA & & NA & & & & \\
\hline Yoon et al. 2014 [32] & & & & & & & NA & NA & & NA & & & & \\
\hline \multicolumn{15}{|c|}{ Uterine sarcoma } \\
\hline Bartosh et al. 2017 [33] & & & & & & & NA & NA & & NA & & & & \\
\hline Tirumani et al. 2014 [34] & & & & & & & NA & NA & & NA & & & & \\
\hline \multicolumn{15}{|c|}{ Vulvar cancer } \\
\hline Prieske et al. 2016 [35] & & & & & & & NA & NA & & NA & & & & \\
\hline \multicolumn{15}{|c|}{ Cervical cancer } \\
\hline Kanayama et al. 2015 [36] & & & & & & & NA & NA & & NA & & & & \\
\hline Kocaer et al. 2018 [37] & & & & & & & NA & NA & & NA & & & & \\
\hline Lin et al. 2018 [38] & & & & & & & NA & NA & & NA & & & & \\
\hline Makino et al. 2016 [39] & & & & & & & NA & NA & & NA & & & & \\
\hline Manders et al. 2018 [40] & & & & & & & NA & NA & & NA & & & & \\
\hline Matsumiya et al. 2016 [41] & & & & & & & NA & NA & & NA & & & & \\
\hline Nartthanarung et al. 2014 [42] & & & & & & & NA & NA & & NA & & & & \\
\hline Sethi et al. 2019 [43] & & & & & & & NA & NA & & NA & & & & \\
\hline Yin et al. 2019 [44] & & & & & & & NA & NA & & NA & & & & \\
\hline Yoon et al. 2013 [45] & & & & & & & NA & NA & & NA & & & & \\
\hline Zhang et al. 2018 [46] & & & & & & & NA & NA & & NA & & & & \\
\hline Zhang et al. 2020 [47] & & & & & & & NA & NA & & NA & & & & \\
\hline Zhou et al. 2020 [48] & & & & & & & NA & NA & & NA & & & & \\
\hline
\end{tabular}

1. Was the research question or objective in this paper clearly stated? 2. Was the study population clearly specified and defined? 3 . Was the participation rate of eligible persons at least $50 \%$ ? 4 . Were all the subjects selected or recruited from the same or similar populations (including the same time period)? Were inclusion and exclusion criteria for being in the study prespecified and applied uniformly to all participants? 5. Was a sample size justification, power description, or variance and effect estimates provided? 6. For the analyses in this paper, was the exposure(s) of interest measured prior to the outcome(s) being measured? 7 . Was the timeframe sufficient so that one could reasonably expect to see an association between exposure and outcome if it existed? 8. For exposures that can vary in amount or level, did the study examine different levels of the exposure as related to the outcome (e.g., categories of exposure, or exposure measured as continuous variable)? 9. Were the exposure measures (independent variables) clearly defined, valid, reliable, and implemented consistently across all study participants? 10. Was the exposure(s) assessed more than once over time? 11. Were the outcome measures (dependent variables) clearly defined, valid, reliable, and implemented consistently across all study participants? 12. Were the outcome assessors blinded to the exposure status of participants? 13. Was loss to follow-up after baseline $20 \%$ or less? 14. Were key potential confounding variables measured and adjusted statistically for their impact on the relationship between exposure(s) and outcome(s)? Yes No, CD, cannot determine; NA, not applicable; NR, not reported. 
Table 2. Characteristics of patients at time of diagnosis of GC bone metastasis in the included studies.

\begin{tabular}{|c|c|c|c|c|c|c|c|c|c|}
\hline Ref. & $\begin{array}{c}\text { Country and } \\
\text { Time Horizon } \\
\text { Covered }\end{array}$ & Type of Study & $\begin{array}{l}\text { Number of } \\
\text { Patients }\end{array}$ & $\begin{array}{c}\text { Bone } \\
\text { Metastases } \\
\text { Occurrence }\end{array}$ & $\begin{array}{l}\text { Median/Mean } \\
\text { Age (Years) }\end{array}$ & $\begin{array}{l}\text { Menopausal } \\
\text { Status }\end{array}$ & $\begin{array}{l}\text { Primary Tumor } \\
\text { Histopathology }\end{array}$ & $\begin{array}{l}\text { Primary Tumor } \\
\text { FIGO Stage } \\
\text { and Grade }\end{array}$ & $\begin{array}{l}\text { Survival/Follow-Up after } \\
\text { Bone Metastasis (Months) }\end{array}$ \\
\hline \multicolumn{10}{|c|}{ Ovarian Cancer } \\
\hline $\begin{array}{c}\text { Deng } 2018 \\
\text { [18] }\end{array}$ & $\begin{array}{c}\text { China } \\
\text { 2010-2014 }\end{array}$ & Retrospective & 1481 & $\begin{array}{l}32 \text { patients } \\
(2.1 \%)\end{array}$ & NR & NR & $\begin{array}{c}\text { Serous and } \\
\text { non-serous ovarian } \\
\text { carcinoma }\end{array}$ & Stage IV & Median OS: 11 months \\
\hline $\begin{array}{l}\text { Sehouli } \\
2013 \\
\text { [19] }\end{array}$ & $\begin{array}{c}\text { Berlin } \\
\text { 1994-2009 }\end{array}$ & Retrospective & 1717 & $\begin{array}{c}26 \text { patients } \\
(1.5 \%)\end{array}$ & $\begin{array}{l}\text { Median age: } \\
54.0\end{array}$ & $\begin{array}{l}\text { Pre-m }(n=11) \\
\text { Post-m }(n=15)\end{array}$ & $\begin{array}{l}\text { Serous }(n=14) \text { and } \\
\text { non-serous }(n=12) \\
\text { ovarian carcinoma }\end{array}$ & $\begin{array}{c}\text { Stage I/II }(n=4) \\
\text { Stage III/IV } \\
(n=22) \\
\text { Grade I/II } \\
(n=9) \\
\text { Grade III } \\
(n=12) \\
\text { Unknown grade } \\
(n=5)\end{array}$ & Median OS: 7.2 months \\
\hline $\begin{array}{c}\text { Zhang } \\
2013 \\
{[20]}\end{array}$ & $\begin{array}{c}\text { China } \\
\text { 2002-2008 }\end{array}$ & Retrospective & 2189 & $\begin{array}{l}26 \text { patients } \\
(1.1 \%)\end{array}$ & Mean age: 46.7 & NR & $\begin{array}{l}\text { Serous ovarian } \\
\text { carcinoma }(n=13) \\
\text { Clear cell ovarian } \\
\text { carcinoma }(n=6) \\
\text { Ovarian germ cell } \\
\text { carcinoma }(n=7)\end{array}$ & $\begin{array}{l}\text { Stage II }(n=3) \\
\text { Stage III/IV } \\
\quad(n=23)\end{array}$ & $\begin{array}{c}\text { Mean survival time: } \\
\text {-No treatment: } 3.0 \text { months } \\
\text {-Disodium pamidronate: } \\
7.0 \text { months } \\
\text {-Chemotherapy: } 8.4 \text { months } \\
\text {-Radionuclide therapy: } \\
11.0 \text { months } \\
\text {-Chemotherapy + } \\
\text { radiotherapy: } 14.2 \text { months } \\
\text {-Chemotherapy }+ \\
\text { radiotherapy +disodium } \\
\text { pamidronate: } 17.3 \text { months } \\
\text {-Chemotherapy }+ \\
\text { radiotherapy }+ \\
\text { radionuclide therapy: } \\
21.5 \text { months }\end{array}$ \\
\hline
\end{tabular}


Table 2. Cont.

\begin{tabular}{|c|c|c|c|c|c|c|c|c|c|}
\hline Ref. & $\begin{array}{c}\text { Country and } \\
\text { Time Horizon } \\
\text { Covered }\end{array}$ & Type of Study & $\begin{array}{l}\text { Number of } \\
\text { Patients }\end{array}$ & $\begin{array}{c}\text { Bone } \\
\text { Metastases } \\
\text { Occurrence }\end{array}$ & $\begin{array}{c}\text { Median/Mean } \\
\text { Age (Years) }\end{array}$ & $\begin{array}{l}\text { Menopausal } \\
\text { Status }\end{array}$ & $\begin{array}{l}\text { Primary Tumor } \\
\text { Histopathology }\end{array}$ & $\begin{array}{c}\text { Primary Tumor } \\
\text { FIGO Stage } \\
\text { and Grade }\end{array}$ & $\begin{array}{l}\text { Survival/Follow-Up after } \\
\text { Bone Metastasis (Months) }\end{array}$ \\
\hline $\begin{array}{l}\text { Zhang } \\
2019 \\
{[21]}\end{array}$ & $\begin{array}{c}\text { China } \\
\text { 2010-2015 }\end{array}$ & Retrospective & 32,178 & $\begin{array}{c}352 \\
(1.0 \%)\end{array}$ & $\begin{array}{c}\text { Mean age: } \\
65.61 \pm 15.12\end{array}$ & NR & $\begin{array}{c}\text { Serous ovarian } \\
\text { carcinoma }(n=87) \\
\text { Non-serous ovarian } \\
\text { carcinoma }(n=205) \\
\text { Unknown ovarian } \\
\text { carcinoma }(n=60)\end{array}$ & $\begin{array}{c}\text { Stage I }(n=23) \\
\text { Stage II }(n=46) \\
\text { Stage III } \\
(n=129) \\
\text { Unknown stage } \\
(n=154) \\
\text { Grade I }(n=4) \\
\text { Grade II }(n=7) \\
\text { Grade III } \\
(n=66) \\
\text { Undifferentiated } \\
(n=34) \\
\text { Unknown grade } \\
(n=241)\end{array}$ & Median OS: 5 months \\
\hline \multicolumn{10}{|c|}{ Endometrial Cancer } \\
\hline $\begin{array}{l}\text { Hong } 2021 \\
\quad[23]\end{array}$ & $\begin{array}{c}\text { USA } \\
\text { 2013-2016 }\end{array}$ & Retrospective & 403 & $\begin{array}{c}18 \\
(4.4 \%)\end{array}$ & Mean age: 66.1 & Post-m & $\begin{array}{l}\text { Endometrial serous } \\
\text { and non-serous } \\
\text { carcinoma }\end{array}$ & Stage IA to IV & Median OS: 16 months \\
\hline $\begin{array}{l}\text { Kimyon } \\
2016[24]\end{array}$ & $\begin{array}{c}\text { Turkey } \\
\text { 1993-2013 }\end{array}$ & Retrospective & 1345 & $\begin{array}{c}10 \\
(0.7 \%)\end{array}$ & Mean age: 61 & Post-m & $\begin{array}{c}\text { Endometrioid } \\
\text { carcinoma } \\
\text { Endometrial serous } \\
\text { carcinoma } \\
\text { Endometrial clear cell } \\
\text { carcinoma } \\
\text { Endometrial } \\
\text { undifferentiated } \\
\text { carcinoma }\end{array}$ & $\begin{array}{l}\text { Stage IB }(n=5) \\
\text { Stage IIIC1 } \\
(n=1) \\
\text { Stage IIIC2 } \\
\quad(n=4) \\
\text { Grade I }(n=3) \\
\text { Grade II }(n=7) \\
\text { Grade III }(n=3)\end{array}$ & $\begin{array}{c}\text { Median OS: } 17.5 \text { months } \\
\text { in patients with Stage IB } \\
\text { and } \\
4 \text { months in } \\
\text { Stage IIIC1-2 }\end{array}$ \\
\hline
\end{tabular}


Table 2. Cont.

\begin{tabular}{|c|c|c|c|c|c|c|c|c|c|}
\hline Ref. & $\begin{array}{c}\text { Country and } \\
\text { Time Horizon } \\
\text { Covered }\end{array}$ & Type of Study & $\begin{array}{l}\text { Number of } \\
\text { Patients }\end{array}$ & $\begin{array}{c}\text { Bone } \\
\text { Metastases } \\
\text { Occurrence }\end{array}$ & $\begin{array}{l}\text { Median/Mean } \\
\text { Age (Years) }\end{array}$ & $\begin{array}{l}\text { Menopausal } \\
\text { Status }\end{array}$ & $\begin{array}{l}\text { Primary Tumor } \\
\text { Histopathology }\end{array}$ & $\begin{array}{c}\text { Primary Tumor } \\
\text { FIGO Stage } \\
\text { and Grade }\end{array}$ & $\begin{array}{l}\text { Survival/Follow-Up after } \\
\text { Bone Metastasis (Months) }\end{array}$ \\
\hline $\begin{array}{l}\text { Li 2020 } \\
\text { [25] }\end{array}$ & $\begin{array}{l}\text { China } \\
\text { 2010-2015 }\end{array}$ & Retrospective & 929 & $\begin{array}{c}36 \\
(3.8 \%)\end{array}$ & $\begin{array}{l}<63 \\
\geq 63\end{array}$ & NR & $\begin{array}{l}\text { Endometrioid and } \\
\text { non-endometrioid } \\
\text { carcinoma }\end{array}$ & Stage IVB & Median OS: 18 months \\
\hline $\begin{array}{c}\text { Liu } 2020 \\
\text { [26] }\end{array}$ & $\begin{array}{l}\text { China } \\
\text { 2010-2014 }\end{array}$ & Retrospective & 2948 & $\begin{array}{c}101 \\
(3.4 \%)\end{array}$ & $\begin{array}{c}\text { From } 47.5 \text { to } \\
52.5\end{array}$ & NR & $\begin{array}{l}\text { Endometrioid and } \\
\text { non-endometrioid } \\
\text { carcinoma }\end{array}$ & $\begin{array}{c}\text { Stage IV } \\
\text { Grade I, II, III }\end{array}$ & Median OS: 6 months \\
\hline $\begin{array}{l}\text { Mao 2020 } \\
{[27]}\end{array}$ & $\begin{array}{l}\text { China } \\
\text { 2010-2015 }\end{array}$ & Retrospective & 69,027 & $\begin{array}{c}388 \\
(n=0.5 \%)\end{array}$ & $\begin{array}{c}<60(n=117) \\
60-80(n=235) \\
<80(n=36)\end{array}$ & NR & $\begin{array}{c}\text { Endometrioid } \\
\text { carcinoma }(n=261) \\
\text { Endometrial serous } \\
(n=46) \text { carcinoma } \\
\text { Endometrial } \\
\text { carcinosarcoma } \\
(n=37) \\
\text { Endometrial clear cell } \\
\text { carcinoma }(n=17) \\
\text { Endometrial mixed } \\
\text { epithelial carcinoma } \\
(n=27)\end{array}$ & $\begin{array}{c}\text { Grade I }(n=41) \\
\text { Grade II } \\
(n=151) \\
\text { Grade III } \\
(n=61) \\
\text { Undifferentiated } \\
(n=117)\end{array}$ & Median OS: 8 months \\
\hline $\begin{array}{l}\text { McEachron } \\
2020[28]\end{array}$ & $\begin{array}{c}\text { USA } \\
\text { 2012-2019 }\end{array}$ & Retrospective & 1085 & $\begin{array}{c}10 \\
(0.9 \%)\end{array}$ & Mean age: 65 & NR & $\begin{array}{c}\text { Endometrioid } \\
\text { carcinoma }(n=7) \\
\text { Endometrial serous } \\
\text { carcinoma }(n=3)\end{array}$ & $\begin{array}{l}\text { Stage I }(n=2) \\
\text { Stage III/IV } \\
\quad(n=8)\end{array}$ & Median OS: 11 months \\
\hline $\begin{array}{l}\text { Ouldamer } \\
2019[29]\end{array}$ & $\begin{array}{c}\text { France } \\
\text { 2001-2013 }\end{array}$ & Retrospective & 1444 & $\begin{array}{c}9 \\
(0.6 \%)\end{array}$ & Mean age: 75.5 & Post-m & $\begin{array}{l}\text { Endometrial } \\
\text { carcinoma }\end{array}$ & $\begin{array}{c}\text { Grade I }(n=1) \\
\text { Grade II }(n=3) \\
\text { Grade III }(n=6)\end{array}$ & Median OS: 15 months \\
\hline $\begin{array}{l}\text { Takeshita } \\
2016[30]\end{array}$ & $\begin{array}{c}\text { Japan } \\
\text { 1995-2014 }\end{array}$ & Retrospective & 926 & $\begin{array}{c}28 \\
(3.0 \%)\end{array}$ & Mean age: 60.5 & Post-m & $\begin{array}{l}\text { Endometrioid and } \\
\text { non-endometrioid car- } \\
\text { cinomaEndometrial } \\
\text { carcinosarcoma }\end{array}$ & $\begin{array}{c}\text { Stage I }(n=3) \\
\text { Stage II }(n=2) \\
\text { Stage III }(n=9) \\
\text { Stage IV }(n=14) \\
\text { Grade I/II } \\
\quad(n=9) \\
\text { Grade III/Others } \\
\quad(n=19)\end{array}$ & Median OS: 6.2 months \\
\hline
\end{tabular}


Table 2. Cont.

\begin{tabular}{|c|c|c|c|c|c|c|c|c|c|}
\hline Ref. & $\begin{array}{c}\text { Country and } \\
\text { Time Horizon } \\
\text { Covered }\end{array}$ & Type of Study & $\begin{array}{l}\text { Number of } \\
\text { Patients }\end{array}$ & $\begin{array}{c}\text { Bone } \\
\text { Metastases } \\
\text { Occurrence }\end{array}$ & $\begin{array}{c}\text { Median/Mean } \\
\text { Age (Years) }\end{array}$ & $\begin{array}{c}\text { Menopausal } \\
\text { Status }\end{array}$ & $\begin{array}{l}\text { Primary Tumor } \\
\text { Histopathology }\end{array}$ & $\begin{array}{l}\text { Primary Tumor } \\
\text { FIGO Stage } \\
\text { and Grade }\end{array}$ & $\begin{array}{l}\text { Survival/Follow-Up after } \\
\text { Bone Metastasis (Months) }\end{array}$ \\
\hline $\begin{array}{l}\text { Uccella } \\
2013[31]\end{array}$ & $\begin{array}{c}\text { USA } \\
1984-2001\end{array}$ & Retrospective & 1632 & $\begin{array}{c}19 \\
(1.1 \%)\end{array}$ & Mean age: 65 & Post-m & $\begin{array}{c}\text { Endometrioid } \\
\text { carcinoma }(n=13) \\
\text { Non-endometrioid } \\
\text { carcinoma }(n=6)\end{array}$ & $\begin{array}{l}\text { Stage I }(n=10) \\
\text { Stage II }(n=1) \\
\text { Stage III }(n=3) \\
\text { Stage IV }(n=5)\end{array}$ & Median OS: 12 months \\
\hline $\begin{array}{c}\text { Yoon } 2014 \\
\text { [32] }\end{array}$ & $\begin{array}{c}\text { South Korea } \\
\text { 1994-2012 }\end{array}$ & Retrospective & 1185 & $\begin{array}{c}21 \\
(1.7 \%)\end{array}$ & Mean age: 59 & NR & $\begin{array}{c}\text { Endometrioid } \\
\text { carcinoma }(n=8) \\
\text { Non-endometrioid } \\
\text { carcinoma }(n=13)\end{array}$ & $\begin{array}{c}\text { Stages II/I } \\
(n=4) \\
\text { Stages III/IV } \\
(n=17) \\
\text { Grade I }(n=1) \\
\text { Grade II }(n=4) \\
\text { Grade III } \\
(n=10) \\
\text { Unknown } \\
(n=6)\end{array}$ & Median OS: 15 months \\
\hline \multicolumn{10}{|c|}{ Uterine Sarcoma } \\
\hline $\begin{array}{l}\text { Tirumani } \\
2014[34]\end{array}$ & $\begin{array}{c}\text { USA } \\
\text { 2000-2012 }\end{array}$ & Retrospective & 113 & $\begin{array}{c}37 \\
(32.7 \%)\end{array}$ & Mean age: 53 & NR & $\begin{array}{c}\text { Uterine } \\
\text { leiomyosarcoma }\end{array}$ & Stage I to IV & Median OS: 45 months \\
\hline \multicolumn{10}{|c|}{ Vulvar Cancer } \\
\hline $\begin{array}{c}\text { Prieske } \\
2016 \\
{[35]}\end{array}$ & $\begin{array}{l}\text { Germany } \\
1996-2013\end{array}$ & Retrospective & 391 & $\begin{array}{c}5 \\
(1.2 \%)\end{array}$ & Median age: 60 & $\begin{array}{l}\text { Pre-m } \\
\text { Post-m }\end{array}$ & $\begin{array}{l}\text { Vulvar squamous-cell } \\
\text { carcinoma }\end{array}$ & $\begin{array}{l}\text { Stage IA/IB } \\
\text { Grade II/III }\end{array}$ & Median OS: 36 months \\
\hline \multicolumn{10}{|c|}{ Cervical Cancer } \\
\hline $\begin{array}{c}\text { Kanayama } \\
2015 \\
{[36]}\end{array}$ & $\begin{array}{c}\text { Japan } \\
\text { 1996-2010 }\end{array}$ & Retrospective & 713 & $\begin{array}{c}37 \\
(5.1 \%)\end{array}$ & Median age: 58 & Post-m & $\begin{array}{c}\text { Cervical } \\
\text { squamous-cell } \\
\text { carcinoma }(n=30) \\
\text { Cervical } \\
\text { non-squamous-cell } \\
\text { carcinoma }(n=7)\end{array}$ & $\begin{array}{c}\text { Stage I/II } \\
\quad(n=18) \\
\text { Stage III/IV } \\
\quad(n=19)\end{array}$ & Median OS: 12 months \\
\hline
\end{tabular}


Table 2. Cont.

\begin{tabular}{|c|c|c|c|c|c|c|c|c|c|}
\hline Ref. & $\begin{array}{l}\text { Country and } \\
\text { Time Horizon } \\
\text { Covered }\end{array}$ & Type of Study & $\begin{array}{l}\text { Number of } \\
\text { Patients }\end{array}$ & $\begin{array}{c}\text { Bone } \\
\text { Metastases } \\
\text { Occurrence }\end{array}$ & $\begin{array}{l}\text { Median/Mean } \\
\text { Age (Years) }\end{array}$ & $\begin{array}{c}\text { Menopausal } \\
\text { Status }\end{array}$ & $\begin{array}{l}\text { Primary Tumor } \\
\text { Histopathology }\end{array}$ & $\begin{array}{l}\text { Primary Tumor } \\
\text { FIGO Stage } \\
\text { and Grade }\end{array}$ & $\begin{array}{l}\text { Survival/Follow-Up after } \\
\text { Bone Metastasis (Months) }\end{array}$ \\
\hline $\begin{array}{c}\text { Kocaer } \\
\text { et al. } 2018 \\
{[37]}\end{array}$ & $\begin{array}{l}\text { Istanbul } \\
\text { 1992-2015 }\end{array}$ & Retrospective & 844 & $\begin{array}{c}18 \\
(2.1 \%)\end{array}$ & $\begin{array}{l}\text { Mean age: } \\
55.8 \pm 10.0\end{array}$ & $\begin{array}{c}\text { Post-m }(n=14), \\
\text { Pre-m }(n=4)\end{array}$ & $\begin{array}{l}\text { Cervical } \\
\text { squamous-cell } \\
\text { carcinoma }\end{array}$ & $\begin{array}{c}\text { Stage I/II }(n=6) \\
\text { Stage III /IV } \\
(n=12) \\
\text { Grade I }(n=1) \\
\text { Grade II }(n=6) \\
\text { Grade III } \\
(n=11)\end{array}$ & $\begin{array}{c}\text { Mean survival: } \\
14.1 \pm 7.8 \text { months }\end{array}$ \\
\hline $\begin{array}{c}\operatorname{Lin} 2019 \\
{[38]}\end{array}$ & $\begin{array}{c}\text { USA } \\
1997-2017\end{array}$ & Retrospective & 1158 & $\begin{array}{c}12 \\
(1.0 \%)\end{array}$ & Median age: 53 & NR & Cervical cancer & $\begin{array}{l}\text { Stage I/II } \\
\text { Stage III/IV }\end{array}$ & Median OS: 7.0 months \\
\hline $\begin{array}{l}\text { Makino } \\
2016[39]\end{array}$ & $\begin{array}{c}\text { Japan } \\
\text { 2000-2010 }\end{array}$ & Retrospective & NR & 75 & Mean age: 52.2 & NR & Cervical cancer & $\begin{array}{c}\text { Stage I/II } \\
(n=40) \\
\text { Stage III/IV } \\
(n=34) \\
\text { Unknown } \\
(n=1)\end{array}$ & $\begin{array}{l}\text { Median OS: } 14 \text { months in } \\
\text { patients with bone } \\
\text { metastases only and } \\
5 \text { months in patients with } \\
\text { extra-osseous metastases }\end{array}$ \\
\hline $\begin{array}{l}\text { Matsumiya } \\
2016[41]\end{array}$ & $\begin{array}{c}\text { Japan } \\
\text { 1995-2014 }\end{array}$ & Retrospective & 925 & $\begin{array}{c}54 \\
(5.8 \%)\end{array}$ & $\begin{array}{l}\text { Median age: } \\
55.5\end{array}$ & $\begin{array}{l}\text { Post-m } \\
\text { Pre-m }\end{array}$ & $\begin{array}{l}\text { Cervical squamous } \\
(n=42) \text { and } \\
\text { non-squamous cell } \\
\text { carcinoma }\end{array}$ & $\begin{array}{l}\text { Stage I/II } \\
\quad(n=21) \\
\text { Stage III/IV } \\
(n=33)\end{array}$ & Median OS: 22 weeks \\
\hline $\begin{array}{l}\text { Nartthanarung } \\
2014 \text { [42] }\end{array}$ & $\begin{array}{l}\text { Thailand } \\
\text { 1998-2010 }\end{array}$ & Retrospective & NR & 68 & $\begin{array}{c}<45 \\
(n=13)>45 \\
(n=39)\end{array}$ & $\begin{array}{l}\text { Post-m } \\
\text { Pre-m }\end{array}$ & Cervical carcinoma & $\begin{array}{c}\text { Stage I/II } \\
(n=28) \\
\text { Stage III/IV } \\
(n=24)\end{array}$ & $\begin{array}{c}\text { Median OS: } \\
<45 \text { years: } 21 \text { months } \\
>45 \text { years: } 34 \text { months }\end{array}$ \\
\hline $\begin{array}{c}\text { Sethi } 2019 \\
\text { [43] }\end{array}$ & $\begin{array}{l}\text { India } \\
2016\end{array}$ & Retrospective & 100 & $\begin{array}{c}11 \\
(11 \%)\end{array}$ & NR & NR & Cervical carcinoma & NR & NR \\
\hline $\begin{array}{c}\text { Yin } 2019 \\
{[44]}\end{array}$ & $\begin{array}{l}\text { China } \\
\text { 2006-2016 }\end{array}$ & Retrospective & 99 & $\begin{array}{c}24 \\
(24.2 \%)\end{array}$ & $\begin{array}{l}\text { Median age: } \\
53\end{array}$ & $\begin{array}{l}\text { Post-m } \\
\text { Pre-m }\end{array}$ & Cervical carcinoma & NR & Median OS: 11.7 months \\
\hline
\end{tabular}


Table 2. Cont.

\begin{tabular}{|c|c|c|c|c|c|c|c|c|c|}
\hline Ref. & $\begin{array}{l}\text { Country and } \\
\text { Time Horizon } \\
\text { Covered }\end{array}$ & Type of Study & $\begin{array}{l}\text { Number of } \\
\text { Patients }\end{array}$ & $\begin{array}{c}\text { Bone } \\
\text { Metastases } \\
\text { Occurrence }\end{array}$ & $\begin{array}{l}\text { Median/Mean } \\
\text { Age (Years) }\end{array}$ & $\begin{array}{c}\text { Menopausal } \\
\text { Status }\end{array}$ & $\begin{array}{l}\text { Primary Tumor } \\
\text { Histopathology }\end{array}$ & $\begin{array}{l}\text { Primary Tumor } \\
\text { FIGO Stage } \\
\text { and Grade }\end{array}$ & $\begin{array}{l}\text { Survival/Follow-Up after } \\
\text { Bone Metastasis (Months) }\end{array}$ \\
\hline $\begin{array}{c}\text { Yoon } 2013 \\
{[45]}\end{array}$ & $\begin{array}{c}\text { Korea } \\
1994-2011\end{array}$ & Retrospective & 2013 & $\begin{array}{c}105 \\
\text { (14 excluded } \\
\text { because of } \\
\text { unavailable } \\
\text { medical } \\
\text { records) } \\
(5.2 \%)\end{array}$ & Mean age: 52.5 & $\begin{array}{l}\text { Post-m } \\
\text { Pre-m }\end{array}$ & Cervical carcinoma & $\begin{array}{c}\text { Stage I/II } \\
(n=37) \\
\text { Stage III/IV } \\
\quad(n=38)\end{array}$ & Median OS: 10 months \\
\hline $\begin{array}{l}\text { Zhang } \\
2018[46]\end{array}$ & $\begin{array}{c}\text { USA } \\
2010-2015\end{array}$ & Retrospective & 19,363 & $\begin{array}{c}469 \\
(2.4 \%)\end{array}$ & $\begin{array}{c}\text { Mean age: } \\
56.43 \pm 13.78\end{array}$ & $\begin{array}{l}\text { Post-m } \\
\text { Pre-m }\end{array}$ & Cervical carcinoma & $\begin{array}{c}\text { Stage I/II } \\
(n=145) \\
\text { Stage III/IV } \\
(n=242) \\
\text { Unknown } \\
(n=82) \\
\text { Grade I } \\
(n=9) \text { Grade II } \\
(n=78) \\
\text { Grade III } \\
(n=211) \\
\text { Unknown } \\
(n=171)\end{array}$ & Median OS: 6 months \\
\hline $\begin{array}{l}\text { Zhang } \\
2020 \text { [47] }\end{array}$ & $\begin{array}{c}\text { China } \\
\text { 2010-2016 }\end{array}$ & Retrospective & 1448 & $\begin{array}{c}520 \\
(35.9 \%)\end{array}$ & $\begin{array}{c}\leq 40: n=72 \\
41-64: n=307 \\
\geq 65 \text { years: } 141\end{array}$ & $\begin{array}{l}\text { Post-m } \\
\text { Pre-m }\end{array}$ & Cervical carcinoma & $\begin{array}{c}\text { Stage IVB } \\
\text { Grade I/II } \\
(n=94) \\
\text { Grade III } \\
(n=241) \\
\text { Unknown } \\
(n=185)\end{array}$ & Median OS: 10 months \\
\hline $\begin{array}{c}\text { Zhou } 2020 \\
{[48]}\end{array}$ & $\begin{array}{c}\text { China } \\
\text { 2010-2016 }\end{array}$ & Retrospective & 1347 & $\begin{array}{c}225 \\
(16.7 \%)\end{array}$ & $\begin{array}{c}\text { Mean age: } \\
57.00 \pm 14.29\end{array}$ & $\begin{array}{l}\text { Post-m } \\
\text { Pre-m }\end{array}$ & Cervical carcinoma & $\begin{array}{c}\text { AJCC T Stage: } \\
\text { T1 }(n=24) \\
\text { T2 }(n=39) \\
\text { T3 }(n=100) \\
\text { T4 }(n=27) \\
\text { TX }(n=35)\end{array}$ & Median OS: 8 months \\
\hline
\end{tabular}


For OC, we selected four retrospectives studies. In these studies, the median survival times after bone metastases diagnosis ranged from 3.0 to 21.5 months [20]. A study by Zhang et al. also reported the mean survival time considering a cohort of patients without GC bone metastases treatment (3.0 months) and a cohort of patients treated with chemotherapy + radiotherapy + radionuclide therapy (21.5 months) [20]. The number of patients enrolled in the studies ranged from 1481 [18] to 32,178 [21], with a bone metastases occurrence that ranged from $1.0 \%$ [21] to $2.1 \%$ [18]. The median patient age at the time of diagnosis of bone metastases ranged from 46.7 [20] to 65.6 [21]. Premenopausal status was reported only in one study, which included $42.3 \%$ premenopausal women [19]. At the time of OC diagnosis, most patients had the International Federation of Gynecology and Obstetrics (FIGO) Stage III/IV with Grade II/III [18-21].

For EC, we founded 11 retrospectives studies. In these studies, the median survival time after bone metastases diagnosis ranged from 4.0 to 18.0 months [24,25], with a study that underlined the difference in median survival between patients with Stage III (4 months) and patients with Stage I (17.5 months). The bone metastases occurrence in this cohort of patients ranged from $0.5 \%$ [27] to $15.4 \%$ [22]; this difference could be due to the different number of enrolled patients, which ranged from 403 [23] to 69,027 in the case of the lowest occurrence [27]. The median patient age at the time of diagnosis of bone metastases ranged from 47.5 [26] to 75.5 [29]. A postmenopausal status was reported in 5/12 studies, while the others did not specify the menopausal status [23,24,29-31]. At the time of EC diagnosis, most patients had a FIGO Stage III-IV with Grade II-III.

For US, we found two retrospectives studies, with a median survival time after bone metastases diagnosis that ranged from 22.0 to 45.0 months [33,34]. Both retrospective studies reported cohort of patients with uterine leiomyosarcoma, with a bone metastases occurrence that ranged from $13.8 \%$ [33] to 32.7\% [34]; the number of enrolled patients varied from 113 [34] to 130 [33]. The median patient age at the time of diagnosis of bone metastases was $\sim 53$ years for both retrospective studies. The postmenopausal status was not specified. At the time of US diagnosis, the FIGO Stage ranged from I to IV.

$\mathrm{VuC}$ bone metastases were detailed in one retrospective study, which described a cohort of 391 patients with an occurrence of bone metastases of $1.2 \%$ [35]. The patients' median age was 60 years, and the study included pre- and postmenopausal women with FIGO Stage I and Grade II-III. All the recruited patients had a squamous-cell VuC with an overall survival after bone metastasis of 36 months.

CC bone metastases represented $\sim 42 \%$ of all studies found with our research strategy, with 13 retrospective studies. These studies showed a median survival time after bone metastases diagnosis that ranged from 7.0 to 34.0 months [38,42]. One study specified an overall survival of 14 months for patients with bone metastases only and a lower overall survival, of 5 months, for patients with extra-osseous metastases [39]. Additionally, Nartthanarung et al. described differences in the overall survival considering the patients mean age, with an overall survival of 21 months in patients $<45$ years and an overall survival of 34 months for patients $>45$ years [42]. Almost all the retrospective studies reported a cohort of patients with squamous-cell cervical cancer, with a bone metastases occurrence that ranged from 1\% [38] to 35.9\% [47] and with cohorts that varied from 99 [44] to 19,363 patients [46]. The median patient age at the time of diagnosis of bone metastases ranged from 40 to 65 years [48]. Most studies included both pre- and postmenopausal women, with a FIGO Stage that ranged from I to IV as well as a Grade that ranged from I to III.

\subsection{Bone Metastases Diagnosis and Main Characteristics}

Bone metastases diagnosis methodology, the length of the bone metastases-free interval, the number and sites of bone metastases, the bone metastases symptoms, and the presence of extraosseous metastases were extracted and are reported in Table 3. 
Table 3. Diagnosis and main characteristics of bone metastases in patients with GCs.

\begin{tabular}{|c|c|c|c|c|c|c|}
\hline Ref. & $\begin{array}{c}\text { Bone } \\
\text { Metastases } \\
\text { Diagnosis } \\
\text { Method }\end{array}$ & $\begin{array}{c}\text { Bone } \\
\text { Metastases- } \\
\text { Free } \\
\text { Interval }\end{array}$ & $\begin{array}{c}\text { Bone } \\
\text { Metastases } \\
\text { Site(s) }\end{array}$ & $\begin{array}{c}\text { Bone } \\
\text { Metastases } \\
\text { Number }\end{array}$ & $\begin{array}{c}\text { Bone } \\
\text { Metastases } \\
\text { Symptoms }\end{array}$ & $\begin{array}{l}\text { Extraosseous } \\
\text { Metastases }\end{array}$ \\
\hline \multicolumn{7}{|c|}{ Ovarian Cancer } \\
\hline Deng 2018 [18] & NR & NR & NR & NR & NR & $23 / 32$ \\
\hline $\begin{array}{c}\text { Sehouli } 2013 \\
\text { [19] }\end{array}$ & $\begin{array}{c}\text { X-ray }(n=16), \\
\text { CT }(n=24) \\
\text { MRI }(n=16), \\
\text { BS }(n=20)\end{array}$ & $\begin{array}{l}12 \text { months } \\
(n=8) \\
>12 \text { months } \\
(n=15) \\
\text { At time of } \\
\text { primary } \\
\text { diagnosis } \\
(n=3)\end{array}$ & $\begin{array}{l}\text { Vertebrae, } \\
\text { pelvic bone, } \\
\text { ribs, bones of } \\
\text { the extremities } \\
\text { and the skull }\end{array}$ & $\begin{array}{l}\text { Multiple: } 21 \\
\text { Single: } 5\end{array}$ & Pain & NR \\
\hline $\begin{array}{c}\text { Zhang } 2013 \\
\text { [20] }\end{array}$ & X-ray, CT, MRI & $\begin{array}{l}\text { Stage I } 3 \text { years } \\
\text { Stage II } 2 \text { years } \\
\text { Stage III } 1 \text { to } \\
5 \text { years } \\
\text { Stage IV: after } \\
\text { diagnosis of } \\
\text { ovarian cancer } \\
\text { and after } 1 \text { year }\end{array}$ & $\begin{array}{c}12 \text { cervical } \\
\text { vertebrae, } \\
10 \text { lumbar } \\
\text { vertebrae, } \\
8 \text { pelvis, } \\
7 \text { thoracic } \\
\text { vertebrae, } \\
5 \text { limbs, } 1 \text { ribs, } \\
2 \text { sternum }\end{array}$ & NR & $\begin{array}{l}\text { Low back pain, } \\
\text { thoracodynia, } \\
\text { difficulty in } \\
\text { walking }\end{array}$ & $\begin{array}{c}9 \text { lungs, } 5 \text { liver, } \\
4 \text { brain, } \\
3 \text { splenic, } \\
2 \text { adrenals, } \\
12 \text { lymphatics }\end{array}$ \\
\hline $\begin{array}{c}\text { Zhang } 2019 \\
\text { [21] }\end{array}$ & NR & NR & NR & NR & NR & $\begin{array}{l}\text { Lung, liver, } \\
\text { brain }\end{array}$ \\
\hline \multicolumn{7}{|c|}{ Endometrial Cancer } \\
\hline Guo 2020 [22] & NR & NR & NR & $\begin{array}{l}\text { Single and } \\
\text { multiple }\end{array}$ & NR & Yes \\
\hline Hong 2021 [23] & $\mathrm{PET} / \mathrm{CT}$ & $6 / 18$ months & $\begin{array}{c}\text { Extremity: } 1 / 18 \\
\text { Axial: } 17 / 18\end{array}$ & $\begin{array}{l}\text { Single and } \\
\text { multiple }\end{array}$ & NR & NR \\
\hline $\begin{array}{c}\text { Kimyon } 2016 \\
{[24]}\end{array}$ & X-ray, CT & 13 months & $\begin{array}{l}\text { Costa, pelvis, } \\
\text { sternum, tibia, } \\
\text { scapula, skull }\end{array}$ & $\begin{array}{c}\text { Single }(n=6) \\
\text { Multiple }(n=4)\end{array}$ & Pain & $n=2$ \\
\hline Li 2020 [25] & CT, X-ray & NR & NR & $\begin{array}{l}\text { Single and } \\
\text { multiple }\end{array}$ & NR & Yes \\
\hline Liu 2020 [26] & NR & NR & NR & $\begin{array}{l}\text { Single and } \\
\text { multiple }\end{array}$ & NR & Yes \\
\hline Mao 2020 [27] & NR & NR & NR & NR & NR & Yes \\
\hline $\begin{array}{c}\text { McEachron } \\
2020 \text { [28] }\end{array}$ & X-ray & $\begin{array}{l}2 \text { patients at } \\
\text { diagnosis } \\
8 \text { patients } \\
14.4 \text { months }\end{array}$ & Vertebrae, hip & $\begin{array}{l}\text { Multiple: } 7 \\
\text { Single: } 3\end{array}$ & NR & Yes \\
\hline $\begin{array}{c}\text { Ouldamer } 2019 \\
{[29]}\end{array}$ & $\begin{array}{c}\text { MRI, CT, } \\
\text { 18-FDG PET CT }\end{array}$ & 19 months & NR & NR & NR & Yes \\
\hline $\begin{array}{c}\text { Takeshita } 2016 \\
\text { [30] }\end{array}$ & $\begin{array}{c}\text { MRI, CT, } \\
\text { 18-FDG PET CT }\end{array}$ & $\begin{array}{l}>12 \text { months: } 12 \\
<12 \text { months: } 16\end{array}$ & $\begin{array}{l}\text { Pelvis, } \\
\text { vertebrae, rib, } \\
\text { clavicle, } \\
\text { scapula, } \\
\text { sternum, skull, } \\
\text { tibia, femur }\end{array}$ & Yes $(n=15)$ & NR & Yes $(n=24)$ \\
\hline
\end{tabular}


Table 3. Cont

\begin{tabular}{|c|c|c|c|c|c|c|}
\hline Ref. & $\begin{array}{c}\text { Bone } \\
\text { Metastases } \\
\text { Diagnosis } \\
\text { Method }\end{array}$ & $\begin{array}{c}\text { Bone } \\
\text { Metastases- } \\
\text { Free } \\
\text { Interval }\end{array}$ & $\begin{array}{c}\text { Bone } \\
\text { Metastases } \\
\text { Site(s) }\end{array}$ & $\begin{array}{c}\text { Bone } \\
\text { Metastases } \\
\text { Number }\end{array}$ & $\begin{array}{c}\text { Bone } \\
\text { Metastases } \\
\text { Symptoms }\end{array}$ & $\begin{array}{c}\text { Extraosseous } \\
\text { Metastases }\end{array}$ \\
\hline $\begin{array}{c}\text { Uccella } 2013 \\
\text { [31] }\end{array}$ & NR & $\begin{array}{l}\text { At diagnosis } \\
\quad(n=3) \\
19.5 \text { months } \\
\quad(n=16)\end{array}$ & $\begin{array}{c}\text { Vertebrae } \\
(44.8 \%), \text { hip } \\
(13.8 \%), \text { skull, } \\
\text { clavicle, } \\
\text { sternum, } \\
\text { humerus, ribs, } \\
\text { femur, leg, } \\
\text { calcaneus }\end{array}$ & $\begin{array}{l}\text { Single }(n=13) \\
\text { and multiple } \\
\quad(n=6)\end{array}$ & $\begin{array}{c}\text { Pain, } \\
\text { inflammation }\end{array}$ & Yes $(n=9)$ \\
\hline Yoon 2014 [32] & PET CT & $\begin{array}{l}\text { At diagnosis } \\
\quad(n=4) \\
9 \text { months } \\
(n=17)\end{array}$ & $\begin{array}{l}\text { Vertebrae, } \\
\text { pelvis, } \\
\text { rib, femur, } \\
\text { acetabulum, } \\
\text { clavicle, } \\
\text { parietal } \\
\text { bone, scapula, } \\
\text { humerus }\end{array}$ & $\begin{array}{l}\text { Single }(n=10) \\
\text { and multiple } \\
\quad(n=11)\end{array}$ & Pain & Yes $(n=13)$ \\
\hline \multicolumn{7}{|c|}{ Uterine Sarcoma } \\
\hline $\begin{array}{c}\text { Bartosh } 2017 \\
\text { [33] }\end{array}$ & NR & 36 months & NR & Single $n=7$ & NR & Yes $(n=11)$ \\
\hline $\begin{array}{c}\text { Tirumani } 2014 \\
{[34]}\end{array}$ & $\mathrm{CT}$ & 7 months & NR & $\begin{array}{l}\text { Single and } \\
\text { multiple }\end{array}$ & NR & Yes \\
\hline \multicolumn{7}{|c|}{ Vulvar Cancer } \\
\hline $\begin{array}{c}\text { Prieske } 2016 \\
\text { [35] }\end{array}$ & X-ray, CT & 13 months & $\begin{array}{c}\text { Vertebrae, } \\
\text { pelvis, thigh }\end{array}$ & $\begin{array}{l}\text { Single and } \\
\text { Multiple }\end{array}$ & NR & $\begin{array}{l}\text { pulmonal and } \\
\text { hepatic }(n=3)\end{array}$ \\
\hline \multicolumn{7}{|c|}{ Cervical Cancer } \\
\hline $\begin{array}{c}\text { Kanayama } 2015 \\
{[36]}\end{array}$ & $\begin{array}{c}\text { X-ray, CT, MRI, } \\
\text { FDG-PET/CT, } \\
\text { BS }\end{array}$ & 10 months & $\begin{array}{l}\text { Pelvis, skull, } \\
\text { vertebrae, rib, } \\
\text { upper and } \\
\text { lower } \\
\text { extremities }\end{array}$ & $\begin{array}{c}\text { Single }(n=25) \\
\text { Multiple } \\
(n=12)\end{array}$ & Pain & Yes $(n=29)$ \\
\hline $\begin{array}{l}\text { Kocaer et al. } \\
2018 \text { [37] }\end{array}$ & CT, MRI, BS & NR & Vertebrae & $\begin{array}{l}\text { Single }(n=7) \\
\text { Multiple } \\
(n=11)\end{array}$ & NR & Yes $(n=8)$ \\
\hline Lin 2018 [38] & FDG-PET & NR & NR & NR & NR & NR \\
\hline $\begin{array}{c}\text { Makino } 2016 \\
\text { [39] }\end{array}$ & NR & $\begin{array}{l}\text { At diagnosis } \\
\quad(n=15)\end{array}$ & $\begin{array}{l}\text { Vertebrae, } \\
\text { pelvis }\end{array}$ & $\begin{array}{c}\text { Single }(n=54) \\
\text { Multiple } \\
(n=18)\end{array}$ & Pain & Yes $(n=43)$ \\
\hline $\begin{array}{c}\text { Manders } 2018 \\
{[40]}\end{array}$ & $\begin{array}{c}\text { CT, PET/CT, } \\
\text { FDG-PET, MRI }\end{array}$ & At diagnosis & Pelvis & $\begin{array}{c}\text { Single }(n=8) \\
\text { Multiple }(n=4)\end{array}$ & Pain & Yes $(n=9)$ \\
\hline $\begin{array}{c}\text { Matsumiya } \\
2016 \text { [41] }\end{array}$ & $\begin{array}{c}\text { CT, PET/CT, } \\
\text { FDG-PET, MRI }\end{array}$ & 11.5 months & $\begin{array}{l}\text { Vertebrae, } \\
\text { pelvis }\end{array}$ & $\begin{array}{c}\text { Single }(n=21) \\
\text { Multiple } \\
(n=33)\end{array}$ & NR & Yes $(n=50)$ \\
\hline $\begin{array}{c}\text { Nartthanarung } \\
2014 \text { [42] }\end{array}$ & $\begin{array}{c}\text { CT, MRI, X-ray, } \\
\text { BS }\end{array}$ & $\begin{array}{c}<45 \text { years: } 16 \\
\text { months } \\
>45 \text { years: } 26 \\
\text { months }\end{array}$ & Pelvis & $\begin{array}{l}\text { Single }(n=7) \\
\text { Multiple } \\
(n=35)\end{array}$ & Pain & Yes $(n=12)$ \\
\hline Sethi 2019 [43] & $\mathrm{CT}$ & NR & NR & NR & NR & Yes \\
\hline Yin 2019 [44] & $\begin{array}{l}\text { MRI, CT, } \\
\text { PET-CT }\end{array}$ & NR & NR & NR & NR & Yes $(n=5)$ \\
\hline
\end{tabular}


Table 3. Cont.

\begin{tabular}{|c|c|c|c|c|c|c|}
\hline Ref. & $\begin{array}{c}\text { Bone } \\
\text { Metastases } \\
\text { Diagnosis } \\
\text { Method }\end{array}$ & $\begin{array}{c}\text { Bone } \\
\text { Metastases- } \\
\text { Free } \\
\text { Interval }\end{array}$ & $\begin{array}{c}\text { Bone } \\
\text { Metastases } \\
\text { Site(s) }\end{array}$ & $\begin{array}{c}\text { Bone } \\
\text { Metastases } \\
\text { Number }\end{array}$ & $\begin{array}{c}\text { Bone } \\
\text { Metastases } \\
\text { Symptoms }\end{array}$ & $\begin{array}{c}\text { Extraosseous } \\
\text { Metastases }\end{array}$ \\
\hline Yoon 2013 [45] & $\begin{array}{l}\text { MRI, CT, } \\
\text { PET-CT }\end{array}$ & 27 months & $\begin{array}{c}\text { Vertebrae, } \\
\text { mandible, tibia }\end{array}$ & $\begin{array}{l}\text { Single }(n=45) \\
\text { Multiple } \\
(n=46)\end{array}$ & Pain & Yes \\
\hline Zhang 2018 [46] & NR & $\begin{array}{l}\text { At diagnosis } \\
\quad(n=364)\end{array}$ & NR & NR & NR & Yes \\
\hline Zhang 2020 [47] & NR & NR & NR & NR & NR & Yes \\
\hline Zhou 2020 [48] & NR & NR & NR & NR & NR & Yes \\
\hline
\end{tabular}

Abbreviations: NR: not reported; X-ray: X-radiography, CT: computed tomography; MRI: magnetic resonance imaging; BS: bone scintigraphy; 18FDG PET-CT: Positron emission tomography with 2-deoxy-2-[fluorine-18]fluoro- D-glucose integrated with computed tomography; PET-CT: Positron emission tomography integrated with computed tomography.

For OC bone metastases, the most common imaging modalities for diagnosing were computed tomography (CT), magnetic resonance imaging (MRI), radiography (X-ray) and, for one study, bone scintigraphy (BS). The bone metastases-free interval ranged from 5 years [20] to 1 year $[19,20]$, in relation to the FIGO stage. In some patients the bone metastases diagnosis was also made at time of the OC primary diagnosis $[19,20]$. In this context, Zhang et al. reported bone metastases diagnosis at the same time of OC primary diagnosis prevalently for patients with FIGO Stage IV [20]. The most reported bone metastases site was the spine $[19,20]$, followed by the pelvic bone, ribs, sternum, bones of the extremities, and skull. Bone metastases were single or multiple, with pain as the main reported symptom $[19,20]$. Extraosseous metastases were frequently present, with the lung, liver, and brain as the main reported organs $[18,20]$.

EC bone metastases were mostly diagnosed with PET/CT or CT alone, followed by MRI and X-ray [22-32]. The bone metastases-free interval ranged from 6 months [23] to 19.5 months [31]. In several patients with EC, the bone metastases diagnosis was made at the time of the primary cancer diagnosis [28,31,32]. Bone metastases sites were both single and multiple, with spine, pelvis, tibia, and femur as the most reported anatomical sites of bone metastases and pain as the most reported bone metastases symptom [28,30-32]. Extraosseous metastases were commonly present, but none of the examined studies specified the site [22-32].

In the studies on US, the bone metastases were diagnosed by $\mathrm{CT}$, showing a bone metastasis-free interval that ranged from 7 months [34] to 3 years [33]. Bone metastases sites were single in one study [33] and both single and multiple in the other study [34], with the presence of extraosseous metastases $[33,34]$.

In the only study on $\mathrm{VuC}$, the bone metastases were diagnosed with $\mathrm{CT}$ and $\mathrm{X}$-ray and presented a bone metastasis-free interval of 13 months [35]. Bone metastases were in single and multiple sites and metastasized to the spine, pelvis, and thigh [35]. Extraosseous metastases were reported in the lungs and liver [35].

For CC bone metastases studies, the most common imaging modalities for diagnosing were $\mathrm{CT}$ and MRI, followed by PET/CT, FDG-PET/CT, X-ray, and BS. The bone metastases-free interval ranged from 10 months [36] to 27 months [45]. However, in most patients, the bone metastases diagnosis was made at time of the primary tumor diagnosis $[39,40,46]$. The main reported bone metastases sites were the spine and pelvis, with bone metastases present in single and multiple sites and with pain as the principal reported symptom [36,37,39-42,45]. Extraosseous metastases were described by almost all studies, but none of them specified the anatomical sites. 


\subsection{Complication of Bone Metastases and Development of Skeletal-Related Events}

Patients with GCs that develop bone metastases prevalently have osteolytic lesions that can lead to complications, referred to as SREs. At present, in these cases the most frequent treatment is to relieve symptoms. The purpose of treatment is to reduce pain, prevent pathological fracture occurrence, inhibit the disease progression, improve function and quality of life, and prolong survival time. Treatment options include comprehensive anti-tumor therapy, i.e., surgery, radiotherapy, chemotherapy, and in some cases bone resorption suppression therapy and other palliative treatments. Complications from bone metastases, i.e., SRE percentage, bone pain, pathological fractures, spinal cord compression, and hypercalcemia, as well as first-line therapies for patients with GC bone metastases are summarized in Table 4.

Table 4. Development of complications and skeletal-related events in patients with GC bone metastases included in the review.

\begin{tabular}{|c|c|c|c|c|c|c|}
\hline Ref. & SREs \% & Bone Pain & $\begin{array}{l}\text { Pathological } \\
\text { Fractures }\end{array}$ & $\begin{array}{l}\text { Spinal Cord } \\
\text { Compression }\end{array}$ & Hypercalcemia & $\begin{array}{c}\text { First-Line Therapy for } \\
\text { Bone Metastases }\end{array}$ \\
\hline \multicolumn{7}{|c|}{ Ovarian Cancer } \\
\hline Deng 2018 [18] & NR & NR & NR & NR & NR & $\begin{array}{c}\text { ChT } \\
\text { Surgery }\end{array}$ \\
\hline Sehouli 2013 [19] & NA & Yes & No & No & NR & ChT, RT \\
\hline Zhang 2013 [20] & $33 \%$ & $n=16$ & $n=8$ & $n=4$ & No & $\begin{array}{c}\text { ChT + RT + surgery } \\
(n=24) \\
\text { Surgery }(n=1)\end{array}$ \\
\hline Zhang 2019 [21] & NR & $n=8$ & Yes & No & NR & $\begin{array}{c}\text { Surgery: } 3 \text { Stage II } \\
\text { Cytoreductive surgery: } \\
13 \text { cases Stage } \\
\text { III and in } 10 \text { cases } \\
\text { Stage IV }\end{array}$ \\
\hline \multicolumn{7}{|c|}{ Endometrial Cancer } \\
\hline Guo 2020 [22] & NR & NR & NR & NR & NR & None \\
\hline Hong 2021 [23] & NR & NR & NR & NR & NR & NR \\
\hline Kimyon 2016 [24] & NR & Yes & NR & NR & NR & ChT, RT, surgery $(n=1)$ \\
\hline Li 2020 [25] & NR & NR & NR & NR & NR & ChT, RT \\
\hline Liu 2020 [26] & NR & NR & NR & NR & NR & Surgery \\
\hline Mao 2020 [27] & NR & NR & NR & NR & NR & NR \\
\hline McEachron 2020 [28] & $1 \%$ & NR & $n=1$ & NR & NR & ChT, RT, \\
\hline Ouldamer 2019 [29] & NR & NR & NR & NR & NR & $\mathrm{RT}$ \\
\hline Takeshita 2016 [30] & NR & NR & No & NR & NR & ChT, RT, surgery \\
\hline Uccella 2013 [31] & NR & Yes & $n=1$ & NR & NR & ChT, RT, surgery \\
\hline Yoon 2014 [32] & NR & $n=17$ & $n=2$ & NR & None & ChT, RT, surgery \\
\hline \multicolumn{7}{|c|}{ Uterine Sarcoma } \\
\hline Bartosh 2017 [33] & NR & NR & NR & NR & NR & ChT, RT, surgery \\
\hline Tirumani 2014 [34] & NR & No & NR & NR & NR & ChT, RT, surgery \\
\hline \multicolumn{7}{|c|}{ Vulvar Cancer } \\
\hline Prieske 2016 [35] & NR & NR & NR & NR & NR & ChT, RT, surgery \\
\hline \multicolumn{7}{|c|}{ Cervical Cancer } \\
\hline Kanayama 2015 [36] & NR & $n=15$ & NR & NR & NR & ChT, RT \\
\hline $\begin{array}{c}\text { Kocaer et al. } 2018 \\
\text { [37] }\end{array}$ & NR & NR & NR & NR & NR & ChT, RT, surgery \\
\hline
\end{tabular}


Table 4. Cont.

\begin{tabular}{|c|c|c|c|c|c|c|}
\hline Ref. & SREs \% & Bone Pain & $\begin{array}{l}\text { Pathological } \\
\text { Fractures }\end{array}$ & $\begin{array}{l}\text { Spinal Cord } \\
\text { Compression }\end{array}$ & Hypercalcemia & $\begin{array}{l}\text { First-Line Therapy for } \\
\text { Bone Metastases }\end{array}$ \\
\hline Lin 2018 [38] & NR & NR & NR & NR & NR & NR \\
\hline Makino 2015 [39] & NR & NR & NR & NR & NR & $\begin{array}{c}\text { ChT, RT, other } \\
\text { palliative treatment }\end{array}$ \\
\hline Manders 2018 [40] & No & Yes & $\begin{array}{c}\text { No } \\
\text { (3 after } \\
\text { radiation) }\end{array}$ & No & $n=3$ & $\begin{array}{c}\text { ChT, RT, other } \\
\text { palliative treatment }\end{array}$ \\
\hline Matsumiya 2016 [41] & No & NR & NR & NR & NR & ChT, RT, surgery \\
\hline $\begin{array}{c}\text { Nartthanarung } 2014 \\
\text { [42] }\end{array}$ & NR & Yes & NR & NR & NR & ChT, RT \\
\hline Sethi 2019 [43] & NR & NR & NR & NR & NR & NR \\
\hline Yin 2019 [44] & NR & NR & NR & NR & NR & $\begin{array}{c}\text { ChT, RT, other } \\
\text { palliative treatment }\end{array}$ \\
\hline Yoon 2013 [45] & NR & Yes & NR & NR & $n=12$ & ChT, RT \\
\hline Zhang 2018 [46] & NR & NR & NR & NR & NR & NR \\
\hline Zhang 2020 [47] & NR & NR & NR & NR & NR & Surgery \\
\hline Zhou 2020 [48] & NR & NR & NR & NR & NR & ChT, RT, surgery \\
\hline
\end{tabular}

Abbreviations: NR: not reported; NA: not applicable; ChT: chemotherapy; RT: radiotherapy.

For OC bone metastases, only $1 / 4$ retrospective studies [19] reported the SRE percentage $(33 \%)$. Bone pain was reported in the majority of the studies $(n=3 / 4)$, while pathological fractures were detected in two studies $[19,20]$. Spinal cord compression was reported only in one study [20], while it was not present in two studies $[19,21]$ and not specified in one study [18]. The absence of hypercalcemia was specified in one study [20], while the other studies did not specify the calcium level. First-line treatments for patients with bone metastases from OC were chemotherapy, radiotherapy, and surgery.

The percentage of SREs for EC bone metastases was reported only in the study by McEachron et al. [28] and was 1\%. Bone pain was reported and specified in three studies $(3 / 11)$, while pathological fractures were reported in $3 / 11$ studies. No studies reported and specified the presence/absence of spinal cord compression as well as data on hypercalcemia. First-line treatments for patients with EC bone metastases were specified in most of the analyzed studies (9/11) and generally were radiotherapy and chemotherapy, followed by surgery.

For US and $\mathrm{VuC}$ bone metastases, data on SREs and the development of bone metastases complications were seldom reported [33-35]. None of the studies on these GC bone metastases reported data on SRE\%, calcium levels, fractures and spinal bone compression, and pathological fractures. First-line treatments for these GC bone metastases were radiotherapy, chemotherapy, and surgery.

None of the 13 retrospective studies on CC bone metastases reported the SRE\%, potential presence of pathological fractures, and spinal cord compression, while two of them reported on hypercalcemia $[40,45]$ and four reported on bone pain $[36,40,42,45]$. Firstline treatments for CC bone metastases were principally radiotherapy and chemotherapy, followed by surgery and other palliative treatments.

\section{Discussion}

Although over the years numerous studies have improved our knowledge on bone metastases secondary to GCs, to date, important gaps are still present due to few relevant reports and a paucity of clinical data. Specifically, there is limited information on clinical datasets, patients' specific characteristics, prognostic and predictive factors among patients, metastatic tumors, and treatments. Additionally, the heterogeneity of GC types/subtypes, 
their histopathology, and the variability across geographical regions (including screening method and missing, underreported, or incorrect data) further complicates the understanding of certain clinical features and specific therapeutic approaches to GC bone metastases. Thus, to better highlight and clarify these aspects, we carried out a review to systematically and qualitatively describe and analyze the clinical characteristics, treatment, and potential contributing and potential prognostic factors of GC bone metastases.

The incidence of GC bone metastases remained at about $1.1-5.2 \%$ for CC, $2-8 \%$ for EC, $1.2 \%$ for OC, and $<1 \%$ for other GCs over decades. However, current methods for primary disease control and the easy accessibility to advanced imaging techniques have increased GC bone metastases incidence in the last 10 years. In our review, the occurrence of GC bone metastases was $1.0-35.9 \%$ for CC, $0.5-15.4 \%$ for EC, $1.0-2.1 \%$ for OC, $13.8-32.7 \%$ for US, and $1.2 \%$ for $\mathrm{VuC}$. Although the lytic component was predominant in these GCs, both processes are usually accelerated within the bone metastasis, resulting in "mixed" lesions in which both lytic and sclerotic components are visible. In fact, autopsy studies revealed that bone metastases can be heterogeneous within a single patient, that is, osteolytic at one site and osteoblastic or mixed at another site [49,50]. This review underlined that GC bone metastases incidence was higher in patients with advanced stage disease (III/IV), though also present in a lower percentage of patients with low tumor stage (I/II) and grade (I). Diagnosis was made mostly via CT scans and X-rays. These methods of diagnosis assess the stromal reaction to the presence of cancer cells within the bone marrow rather than depicting the cancer foci themselves. This lack of direct depiction of tumor foci limits early metastatic detection and assessment of the response of bone metastases to treatment. In fact, to limit this problem and to improve the assessment of GC metastatic bone disease, several studies used high-sensitivity imaging methods such as a PET scan, with various radiotracers, and whole-body MRIs [51]. Once bone metastasis has been identified, the treatment is frequently multimodal and interdisciplinary. In this review, the treatment modalities most often used for GC bone metastases were chemotherapy and radiotherapy. The treatment of general pain was the common factor among all GC types, but instability, bone fractures, and spinal cord compression were also present. In these cases, bisphosphonates (which are characterized by ease of administration, long duration of action, safety, and effectiveness) such as zoledronic acid, alendronate, risedronate, and the third-generation agent ibandronate, or RANKL inhibitor, i.e., denosumab, were also used to reduce the incidence of fractures or spinal cord compressions and to relieve diffuse pain [52]. In addition, to relieve suffering and provide the best possible quality of life for both patients and their families, no matter what the treatment course, palliative care (i.e., nutrition, rehabilitation, control of symptoms such as pain, dyspnea, fear, and anxiety) was also used in addition to chemotherapy and radiotherapy $[53,54]$. Good palliative care preserves the patient's quality of life through a predominantly multidimensional approach to symptom control of evolving multi-morbidity and side effects of the primary treatment. In most studies, surgical intervention was also carried out, mostly to manage the structural complications associated with bone destruction and/or nerve compression. However, despite these interventions, the patient's overall survival after bone metastasis diagnosis and treatments was poor and coincided with previous research findings. The longest median overall survival time found in this review was 45 months; however, most studies indicate a median overall survival of $\sim 12$ months. In agreement with the literature, our review revealed that GC bone metastases most affected the axial skeleton and in particular the vertebral column [13]. The causes of this trend are not yet fully understood, but it has been hypothesized that the cellular and molecular characteristics of cancer cells and the tissues to which they metastasize are critical and affect the pattern of metastatic spread; however contradictory or inconclusive results have been reported [14,55]. As shown by this review, these conflicting results can likely be explained by the high heterogeneity in patient populations, cancer treatment, and study methodology. Interestingly, our review showed that most patients with GC bone metastases were in menopause; thus, it is possible to speculate that menopausal status can have an influence on the development of GC bone metastases, confirming the protective 
influence of estrogen on bone density. This aspect was also confirmed for breast cancer bone metastases, in which bimodal interactions between pre-existing estrogen deficiency due to osteoporosis (and related factors) and bone metastasis development was detected. Looking at the literature, it is clear that bone metastases and estrogen deficiency present numerous sharing factors, e.g., disorders in monocyte and macrophage functions and consequent alterations in immune functions, alterations in the balance between pro- and anti-inflammatory regulators, improvement in angiogenesis, platelet deregulation, thromboembolism events, extracellular matrix components, and hormone changes [14]. However, several other contributing factors, such as specific cellular and molecular alterations, gene signature changes, and specific relationships in bone remodeling and primary tumor cells, could further influence the development of GC bone metastases, also considering that bone colonization by malignant cells includes complex interplays between tumor cells and resident bone cells (i.e., osteoclasts, osteoblasts, and osteocytes), bone marrow cells, and the bone matrix. These aspects also suggest that effective treatment strategies for bone metastasis should consider an association of bone-targeted agents in combination with different local and/or systemic anti-tumor strategies for the primary tumor. Thus, a multidisciplinary approach involving oncologists, radiotherapists, orthopedic surgeons, intervention radiologists, pain specialists, and palliative care physicians is mandatory. Furthermore, other specific drawbacks of existing clinical treatments, e.g., systemic administration of antiresorptive drugs and/or anti-tumor agents and/or radiopharmaceuticals, involve adverse effects of these treatments on normal bone metabolism, which may result in harmful outcomes for treated patients. Thus, new and advanced therapeutic options are needed. In this context, emerging bone-targeting therapies are based on the use of anabolic agents or molecules with a dual action, i.e., therapies able to activate osteoblasts and inhibit osteoclasts, or on specific drugs able to target the bone microenvironment. Other promising targets comprise molecules such as small non-coding RNAs (miRNAs), which function as key regulators of various biological and pathological processes, including physiological bone remodeling and bone metastasis. Several studies evaluated the efficacy of these miRNAs as antimetastatic agents and/or as predictors of metastatic bone disease. In detail, recent studies provided evidence that miR-338-3p, miR-208a-5p, $\mathrm{miR}-4443$, and miR-5195-3p contribute to metastasis and GC tumorigenesis via multiple mechanisms [56-58]. Furthermore, several authors also evaluated circulating tumor DNA (ctDNA) and circulating tumor cells (CTCs) as alternative "liquid biopsies" modalities in patients with GC metastases [59-61]. However, the use of cell-free DNA (cfDNA) could be associated with the prediction of response to targeted therapy and the detection of subpopulations/gene signatures of cfDNA, allowing diagnosis, stratification of therapy, and more accurate prognosis. Despite this wide platform of strategies and approaches, their use in the clinical setting remains limited since their application would require a multistage procedure with multiannual and toilsome research approaches. In this context, the development of specific algorithms through recent technological advances related to artificial intelligence could offer a potential alternative and contribution to analyze panels of specific markers/parameters associated with imaging data to determine individual risk factors for GC bone metastases, thus triggering the use of therapies/treatments able to increase the bone-disease-free interval and the survival time after bone metastases.

Our review has several limitations. First, the retrospective nature of the analyzed studies makes them subject to selection bias. Second, most of the included studies had a limited sample size (only 8/31 studies included had more than 100 patients with GC bone metastases), thus lacking sufficient statistical power. Third, there was a marked heterogeneity in patient populations. And finally, methods for detecting and characterizing GC bone metastases complications and skeletal-related events are often not reported.

In conclusion, the results of this systematic review give the first dataset for a greater understanding of GC bone metastases that could be able to guide clinicians towards a more "personalized" patient management. Obviously, further well-designed clinical studies able to accurately determine the risk of developing bone metastases after a diagnosis of GCs are 
mandatory. We look forward to future prospective and large population-based research on this complex issue, which we hope will improve and increase quality of life and patient survival time.

\section{Materials and Methods}

\subsection{Eligibility Criteria}

The PICOS framework (population, intervention, comparison, outcomes, study design) was used to formulate the questions for this study: (1) patients with bone metastases from GCs, i.e., OC, EC, US, VuC, VaC, CC (Population); (2) not applicable (Interventions); (3) not applicable (Comparisons); (4) studies that reported incidence, complication (e.g., skeletal-related events), and prognostic characteristics (age, primary tumor aggressiveness, e.g., subtype, stage, grade, etc.) of bone metastases in patients with GCs (Outcomes); and (4) cohort studies, including randomized controlled trials (Study Design). The focused question was "What are the clinical characteristics, treatment, and potential contributing and prognostic factors of bone metastases from GCs in the clinical setting?". Studies from 24 May 2011 to 19 August 2021 were included in this review if they met the PICOS criteria.

We excluded studies investigating (1) only primary GCs, (2) metastatic sites different than bone, (3) cancers other than bone metastatic GCs, (4) different types of GC bone metastases, considered all together. Additionally, we excluded case reports, case series, abstracts, editorials, letters, comments to the editor, reviews, meta-analyses, book chapters, and articles not written in English.

\subsection{Information Source and Search Strategies}

Our literature review involved a systematic search conducted on 19 August 2021. We performed our review according to the PRISMA 2020 statement [62]. The search was carried out on PubMed, Scopus, Web of Science Core Collection, and Cochrane Central Register of Controlled Trials databases to identify studies on bone metastases from GCs, specifically OC, EC, US, $\mathrm{VuC}, \mathrm{VaC}$, and CC. A search was conducted combining the terms "bone metastasis" AND "ovarian cancer", "bone metastasis" AND "endometrial cancer", "bone metastasis" AND "uterine sarcoma”, "bone metastasis" AND "vulvar cancer", "bone metastasis" AND "vaginal cancer", "bone metastasis" AND "cervical cancer"; for each of these terms, free words and controlled vocabulary specific to each bibliographic database were combined using the operator "OR". The combination of free vocabulary and/or Medical Subject Heading (MeSH) terms for the identification of studies in PubMed, Scopus, Web of Science Core Collection, and Cochrane Central Register of Controlled Trials are reported in Table 5 .

Table 5. Search terms used for PubMed, Scopus, Web of Science Core Collection, and ClinicalTrials.gov.

\begin{tabular}{|c|c|c|}
\hline Database & Search Term & Free Vocabulary and/or Medical Subject Headings (MeSH) Terms \\
\hline \multirow{2}{*}{ PubMed } & $\begin{array}{l}\text { "bone metastasis" } \\
\text { AND } \\
\text { "ovarian cancer" }\end{array}$ & $\begin{array}{l}\text { ("bone and bones"[MeSH Terms] OR ("bone"[All Fields] AND "bones"[All Fields]) OR } \\
\text { "bone and bones"[All Fields] OR "bone"[All Fields]) AND ("metastasi"[All Fields] OR } \\
\text { "neoplasm metastasis"[MeSH Terms] OR ("neoplasm"[All Fields] AND "metastasis"[All } \\
\text { Fields]) OR "neoplasm metastasis"[All Fields] OR "metastasis"[All Fields]) AND } \\
\text { ("ovarian neoplasms"[MeSH Terms] OR ("ovarian"[All Fields] AND "neoplasms"[All } \\
\text { Fields]) OR "ovarian neoplasms"[All Fields] OR ("ovarian"[All Fields] AND "cancer"[All } \\
\text { Fields]) OR "ovarian cancer"[All Fields]) AND ("2011/05/24"[PDAT]: } \\
\text { "2021/05/24"[PDAT]) }\end{array}$ \\
\hline & $\begin{array}{l}\text { "bone metastasis" } \\
\text { AND } \\
\text { "endometrial cancer" }\end{array}$ & $\begin{array}{l}\text { ("bone and bones"[MeSH Terms] OR ("bone"[All Fields] AND “bones"[All Fields]) OR } \\
\text { "bone and bones"[All Fields] OR "bone"[All Fields]) AND ("metastasi"[All Fields] OR } \\
\text { "neoplasm metastasis"[MeSH Terms] OR ("neoplasm"[All Fields] AND "metastasis"[All } \\
\text { Fields]) OR "neoplasm metastasis"[All Fields] OR "metastasis"[All Fields]) AND } \\
\text { ("endometrial neoplasms"[MeSH Terms] OR ("endometrial"[All Fields] AND } \\
\text { "neoplasms"[All Fields]) OR “endometrial neoplasms"[All Fields] OR ("endometrial"[All } \\
\text { Fields] AND “cancer"[All Fields]) OR “endometrial cancer"[All Fields]) AND } \\
\text { ("2011/05/24"[PDAT]: "2021/05/24"[PDAT]) }\end{array}$ \\
\hline
\end{tabular}


Table 5. Cont.

\begin{tabular}{|c|c|c|}
\hline Database & Search Term & Free Vocabulary and/or Medical Subject Headings (MeSH) Terms \\
\hline & $\begin{array}{l}\text { "bone metastasis" } \\
\text { AND } \\
\text { "uterine sarcoma" }\end{array}$ & $\begin{array}{l}\text { ("bone and bones"[MeSH Terms] OR ("bone"[All Fields] AND "bones"[All Fields]) OR } \\
\text { "bone and bones"[All Fields] OR "bone"[All Fields]) AND ("metastasi"[All Fields] OR } \\
\text { "neoplasm metastasis"[MeSH Terms] OR ("neoplasm"[All Fields] AND "metastasis"[All } \\
\text { Fields]) OR "neoplasm metastasis"[All Fields] OR "metastasis"[All Fields]) AND } \\
\text { (("uterin"[All Fields] OR "uterines"[All Fields] OR "uterus"[MeSH Terms] OR } \\
\text { "uterus"[All Fields] OR "uterine"[All Fields]) AND ("sarcoma"[MeSH Terms] OR } \\
\text { "sarcoma"[All Fields] OR "sarcomas"[All Fields] OR "sarcoma s"[All Fields])) AND } \\
\text { ("2011/05/24"[PDAT]: "2021/05/24"[PDAT]) }\end{array}$ \\
\hline & $\begin{array}{l}\text { "bone metastasis" } \\
\text { AND } \\
\text { "vulvar cancer" }\end{array}$ & $\begin{array}{l}\text { ("bone and bones"[MeSH Terms] OR ("bone"[All Fields] AND "bones"[All Fields]) OR } \\
\text { "bone and bones"[All Fields] OR "bone"[All Fields]) AND ("metastasi"[All Fields] OR } \\
\text { "neoplasm metastasis"[MeSH Terms] OR ("neoplasm"[All Fields] AND "metastasis"[All } \\
\text { Fields]) OR "neoplasm metastasis"[All Fields] OR "metastasis"[All Fields]) AND ("vulvar } \\
\text { neoplasms"[MeSH Terms] OR ("vulvar"[All Fields] AND "neoplasms"[All Fields]) OR } \\
\text { "vulvar neoplasms"[All Fields] OR ("vulvar"[All Fields] AND "cancer"[All Fields]) OR } \\
\text { "vulvar cancer"[All Fields]) AND ("2011/05/24"[PDAT]: "2021/05/24"[PDAT]) }\end{array}$ \\
\hline & $\begin{array}{l}\text { "bone metastasis" } \\
\text { AND } \\
\text { "vaginal cancer" }\end{array}$ & $\begin{array}{l}\text { ("bone and bones"[MeSH Terms] OR ("bone"[All Fields] AND "bones"[All Fields]) OR } \\
\text { "bone and bones"[All Fields] OR "bone"[All Fields]) AND ("metastasi"[All Fields] OR } \\
\text { "neoplasm metastasis"[MeSH Terms] OR ("neoplasm"[All Fields] AND "metastasis"[All } \\
\text { Fields]) OR "neoplasm metastasis"[All Fields] OR "metastasis"[All Fields]) AND } \\
\text { ("vaginal neoplasms"[MeSH Terms] OR ("vaginal"[All Fields] AND “neoplasms"[All } \\
\text { Fields]) OR "vaginal neoplasms"[All Fields] OR ("vaginal"[All Fields] AND "cancer"[All } \\
\text { Fields]) OR "vaginal cancer"[All Fields]) AND ("2011/05/24"[PDAT]: } \\
\text { "2021/05/24"[PDAT]) }\end{array}$ \\
\hline & $\begin{array}{l}\text { "bone metastasis" } \\
\text { AND } \\
\text { "cervical cancer" }\end{array}$ & 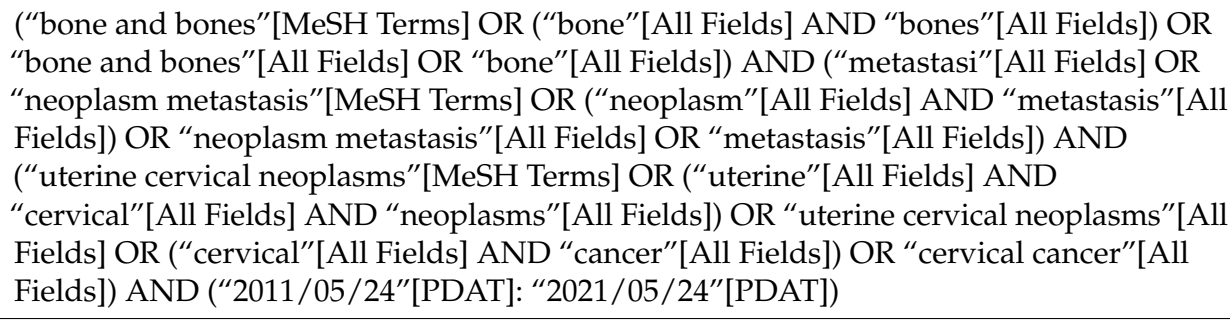 \\
\hline \multirow{6}{*}{$\begin{array}{l}\text { Web of } \\
\text { Science } \\
\text { Core } \\
\text { Collection }\end{array}$} & $\begin{array}{l}\text { bone metastasis" } \\
\text { AND } \\
\text { "ovarian cancer" }\end{array}$ & $\begin{array}{l}\text { (TS = bone metastasis OR TS = bone neoplasm metastasis) AND }(\mathrm{TS}=\text { ovarian cancer OR } \\
\text { TS = ovarian neoplasms } \text { - with Publication Year from } 2011 \text { to } 2021\end{array}$ \\
\hline & $\begin{array}{l}\text { "bone metastasis" } \\
\text { AND } \\
\text { "endometrial cancer" }\end{array}$ & $\begin{array}{l}\text { (TS = bone metastasis OR TS = bone neoplasm metastasis) AND (TS = endometrial cancer } \\
\text { OR TS = endometrial neoplasms)—with Publication Year from } 2011 \text { to } 2021\end{array}$ \\
\hline & $\begin{array}{l}\text { "bone metastasis" } \\
\text { AND } \\
\text { "uterine sarcoma" }\end{array}$ & $\begin{array}{l}\text { (TS = bone metastasis OR TS = bone neoplasm metastasis) AND (TS = uterine sarcoma } \\
\text { OR TS = uterin sarcoma) — with Publication Year from } 2011 \text { to } 2021\end{array}$ \\
\hline & $\begin{array}{l}\text { "bone metastasis" } \\
\text { AND } \\
\text { "vulvar cancer" }\end{array}$ & $\begin{array}{l}(\mathrm{TS}=\text { bone metastasis OR TS = bone neoplasm metastasis }) \text { AND }(\mathrm{TS}=\text { vulvar cancer OR } \\
\text { TS = vulvar neoplasms }) \text { - with Publication Year from } 2011 \text { to } 2021\end{array}$ \\
\hline & $\begin{array}{l}\text { "bone metastasis" } \\
\text { AND } \\
\text { "vaginal cancer" }\end{array}$ & $\begin{array}{l}\text { (TS = bone metastasis OR TS = bone neoplasm metastasis) AND }(\mathrm{TS}=\text { vaginal cancer OR } \\
\text { TS = vaginal neoplasms } \text { — with Publication Year from } 2011 \text { to } 2021\end{array}$ \\
\hline & $\begin{array}{l}\text { "bone metastasis" } \\
\text { AND } \\
\text { "cervical cancer" }\end{array}$ & $\begin{array}{l}\text { (TS = bone metastasis OR TS = bone neoplasm metastasis }) \text { AND }(\text { TS = cervical cancer OR } \\
\text { TS = cervical neoplasms } \text { - with Publication Year from } 2011 \text { to } 2021\end{array}$ \\
\hline
\end{tabular}


Table 5. Cont.

\begin{tabular}{|c|c|c|}
\hline Database & Search Term & Free Vocabulary and/or Medical Subject Headings (MeSH) Terms \\
\hline \multirow{6}{*}{ Scopus } & $\begin{array}{l}\text { bone metastasis" } \\
\text { AND } \\
\text { "ovarian cancer" }\end{array}$ & $\begin{array}{l}\text { (TITLE-ABS-KEY (bone AND metastasis) OR TITLE-ABS-KEY (bone AND neoplasm } \\
\text { AND metastasis) AND TITLE-ABS-KEY (ovarian AND cancer) OR TITLE-ABS-KEY } \\
\text { (ovarian AND neoplasms)) AND PUBYEAR > } 2010\end{array}$ \\
\hline & $\begin{array}{l}\text { "bone metastasis" } \\
\text { AND } \\
\text { "endometrial cancer" }\end{array}$ & $\begin{array}{l}\text { (TITLE-ABS-KEY (bone AND metastasis) OR TITLE-ABS-KEY (bone AND neoplasm } \\
\text { AND metastasis) AND TITLE-ABS-KEY (endometrial AND cancer) OR TITLE-ABS-KEY } \\
\text { (endometrial AND neoplasms)) AND PUBYEAR > } 2010\end{array}$ \\
\hline & $\begin{array}{l}\text { "bone metastasis" } \\
\text { AND } \\
\text { "uterine sarcoma" }\end{array}$ & $\begin{array}{l}\text { (TITLE-ABS-KEY (bone AND metastasis) OR TITLE-ABS-KEY (bone AND neoplasm } \\
\text { AND metastasis) AND TITLE-ABS-KEY (uterine AND sarcoma) OR TITLE-ABS-KEY } \\
\text { (uterin AND sarcoma)) AND PUBYEAR > } 2010\end{array}$ \\
\hline & $\begin{array}{l}\text { "bone metastasis" } \\
\text { AND } \\
\text { "vulvar cancer" }\end{array}$ & $\begin{array}{l}\text { (TITLE-ABS-KEY (bone AND metastasis) OR TITLE-ABS-KEY (bone AND neoplasm } \\
\text { AND metastasis) AND TITLE-ABS-KEY (vulvar AND cancer) OR TITLE-ABS-KEY } \\
\text { (vulvar AND neoplasms)) AND PUBYEAR > } 2010\end{array}$ \\
\hline & $\begin{array}{l}\text { "bone metastasis" } \\
\text { AND } \\
\text { "vaginal cancer" }\end{array}$ & $\begin{array}{l}\text { (TITLE-ABS-KEY (bone AND metastasis) OR TITLE-ABS-KEY (bone AND neoplasm } \\
\text { AND metastasis) AND TITLE-ABS-KEY (vaginal AND cancer) OR TITLE-ABS-KEY } \\
\text { (vaginal AND neoplasms)) AND PUBYEAR > } 2010\end{array}$ \\
\hline & $\begin{array}{l}\text { "bone metastasis" } \\
\text { AND } \\
\text { "cervical cancer" }\end{array}$ & $\begin{array}{l}\text { (TITLE-ABS-KEY (bone AND metastasis) OR TITLE-ABS-KEY (bone AND neoplasm } \\
\text { AND metastasis) AND TITLE-ABS-KEY (cervical AND cancer) OR TITLE-ABS-KEY } \\
\text { (cervical AND neoplasms)) AND PUBYEAR > } 2010\end{array}$ \\
\hline \multirow{6}{*}{$\begin{array}{l}\text { Cochrane } \\
\text { Central } \\
\text { Register of } \\
\text { Controlled } \\
\text { Trials }\end{array}$} & $\begin{array}{l}\text { bone metastasis" } \\
\text { AND } \\
\text { "ovarian cancer" }\end{array}$ & $\begin{array}{l}\text { ovarian cancer in Title Abstract Keyword AND bone metastasis in Title Abstract } \\
\text { Keyword-with Cochrane Library publication date Between May } 2011 \text { and May } 2021 \\
\text { (Word variations have been searched) }\end{array}$ \\
\hline & $\begin{array}{l}\text { "bone metastasis" } \\
\text { AND } \\
\text { "endometrial cancer" }\end{array}$ & $\begin{array}{l}\text { endometrial cancer in Title Abstract Keyword AND bone metastases in Title Abstract } \\
\text { Keyword-with Cochrane Library publication date Between May } 2011 \text { and May } 2021 \\
\text { (Word variations have been searched) }\end{array}$ \\
\hline & $\begin{array}{l}\text { "bone metastasis" } \\
\text { AND } \\
\text { "uterine sarcoma" }\end{array}$ & $\begin{array}{l}\text { uterine sarcoma in Title Abstract Keyword AND bone metastases in Title Abstract } \\
\text { Keyword-with Cochrane Library publication date Between May } 2011 \text { and May } 2021 \\
\text { (Word variations have been searched) }\end{array}$ \\
\hline & $\begin{array}{l}\text { "bone metastasis" } \\
\text { AND } \\
\text { "vulvar cancer" }\end{array}$ & $\begin{array}{l}\text { vulvar cancer in Title Abstract Keyword AND bone metastases in Title Abstract } \\
\text { Keyword-with Cochrane Library publication date Between May } 2011 \text { and May } 2021 \\
\text { (Word variations have been searched) }\end{array}$ \\
\hline & $\begin{array}{l}\text { "bone metastasis" } \\
\text { AND } \\
\text { "vaginal cancer" }\end{array}$ & $\begin{array}{l}\text { vaginal cancer in Title Abstract Keyword AND bone metastases in Title Abstract } \\
\text { Keyword-with Cochrane Library publication date Between May } 2011 \text { and May } 2021 \\
\text { (Word variations have been searched) }\end{array}$ \\
\hline & $\begin{array}{l}\text { "bone metastasis" } \\
\text { AND } \\
\text { "cervical cancer" }\end{array}$ & $\begin{array}{l}\text { cervical cancer in Title Abstract Keyword AND bone metastases in Title Abstract } \\
\text { Keyword-with Cochrane Library publication date Between May } 2011 \text { and May } 2021 \\
\text { (Word variations have been searched) }\end{array}$ \\
\hline
\end{tabular}

\subsection{Study Selection and Data Extraction}

Possible relevant articles were screened, using title and abstract, by two reviewers, and articles that did not meet the inclusion criteria were excluded. After screening the title and abstract, articles were submitted to a public reference manager to eliminate duplicates. Subsequently, the remaining full-text articles were retrieved and examined by two reviewers. Any disagreement was resolved through discussion until a consensus was reached or with the involvement of a third reviewer.

Data from the retrieved studies were tabulated taking into consideration the studies' general characteristics, the GC bone metastases diagnosis and main characteristics, and the development of complications, e.g., skeletal-related events, in GCs bone metastases.

\subsection{Assessment of Methodological Quality}

Two reviewers independently assessed the methodological quality of selected studies. In case of disagreement, they attempted to reach consensus; if this failed, a third 
reviewer made the final decision. The methodological quality of cohort studies was assessed using the Quality Assessment Tools of the National Heart, Lung, and Blood Institute (NHLBI) $[16,17]$.

Author Contributions: Conceptualization, F.S. and M.F.; methodology, F.S. and A.M.P.; data curation, F.S., A.M.P. and D.C.; writing—original draft preparation, F.S., A.M.P., D.C., V.B., A.G., S.T., P.D.I. and M.F.; writing-review and editing, F.S., A.M.P., D.C., V.B., A.G., S.T., P.D.I. and M.F.; supervision, M.F. All authors have read and agreed to the published version of the manuscript.

Funding: This research received no external funding.

Institutional Review Board Statement: Not applicable.

Informed Consent Statement: Not applicable.

Data Availability Statement: Not applicable.

Acknowledgments: This work was supported by IRCCS Istituto Ortopedico Rizzoli (Ricerca Corrente).

Conflicts of Interest: The authors declare no conflict of interest.

\section{References}

1. Coleman, R.; Body, J.J.; Aapro, M.; Hadji, P.; Herrstedt, J.; ESMO Guidelines Working Group. Bone health in cancer patients: ESMO Clinical Practice Guidelines. Ann. Oncol. 2014, 25, iii124-iii37. [CrossRef]

2. Dowdy, S.C.; Cliby, W.A.; Famuyide, A.O. Quality indicators in gynecologic oncology. Gynecol. Oncol. 2018, 151, 366-373. [CrossRef]

3. Jemal, A.; Siegel, R.; Xu, J.; Ward, E. Cancer statistics, 2010. CA Cancer J. Clin. 2010, 60, 277-300. [CrossRef]

4. Ferlay, J.; Parkin, D.M.; Steliarova-Foucher, E. Estimates of cancer incidence and mortality in Europe in 2008. Eur. J. Cancer 2010, 46, 765-781. [CrossRef] [PubMed]

5. Kehoe, S.M.; Zivanovic, O.; Ferguson, S.E.; Barakat, R.R.; Soslow, R.A. Clinicopathologic features of bone metastases and outcomes in patients with primary endometrial cancer. Gynecol. Oncol. 2010, 117, 229-233. [CrossRef]

6. Mariani, A.; Webb, M.J.; Keeney, G.L.; Calori, G.; Podratz, K.C. Hematogenous dissemination in corpus cancer. Gynecol. Oncol. 2001, 80, 233-238. [CrossRef] [PubMed]

7. Lengyel, E. Ovarian cancer development and metastasis. Am. J. Pathol. 2010, 177, 1053-1064. [CrossRef] [PubMed]

8. Thanapprapasr, D.; Nartthanarung, A.; Likittanasombut, P.; Ayudhya, N.I.N.; Charakorn, C.; Udomsubpayakul, U.; Subhadarbandhu, T.; Wilailak, S. Bone metastasis in cervical cancer patients over a 10-year period. Int. J. Gynecol. Cancer 2010, 20, 373-378. [CrossRef] [PubMed]

9. Cormio, G.; Rossi, C.; Cazzolla, A.; Resta, L.; Loverro, G.; Greco, P.; Selvaggi, L. Distant metastases in ovarian carcinoma. Int. J. Gynecol. Cancer 2003, 13, 125-129. [CrossRef]

10. Descamps, P.; Calais, G.; Moire, C.; Bertrand, P.; Castiel, M.; Le Floch, O.; Lansac, J.; Body, G. Predictors of distant recurrence in clinical stage I or II endometrial carcinoma treated by combination surgical and radiation therapy. Gynecol. Oncol. 1997, 64, 54-58. [CrossRef]

11. Ghosh, S.; Kotne, S.; Nayak, S.R.; Turlapati, S.P.V. Vulvar cancer with bone metastases: A rare case report. IJCRR 2013, 5, 49-52.

12. Dutka, J.; Sosin, P.; Urban, M. Efficacy of operative treatment for pathological fractures in bone metastases in relation to length and comfort of survival. Chir. Narzadow Ruchu Ortop. Pol. 2000, 65, 643-649. [PubMed]

13. Liu, S.; Zhou, X.; Song, A.; Huo, Z.; Yao, S.; Wang, Y.; Liu, Y.; Pan, L.Y. Clinical Characteristics and Prognostic Analysis of Gynecologic Cancer with Spinal Metastases: A Single-Center Retrospective Study. Cancer Manag. Res. 2020, 12, 7515-7525. [CrossRef]

14. Fornetti, J.; Welm, A.L.; Stewart, S.A. Understanding the Bone in Cancer Metastasis. J. Bone Miner Res. 2018, 33, 2099-2113. [CrossRef] [PubMed]

15. Falicov, A.; Fisher, C.G.; Sparkes, J.; Boyd, M.C.; Wing, P.C.; Dvorak, M.F. Impact of surgical intervention on quality of life in patients with spinal metastases. Spine 2006, 31, 2849-2856. [CrossRef] [PubMed]

16. Study Quality Assessment Tools. Available online: https://www.nhlbi.nih.gov/health-topics/study-quality-assessment-tools (accessed on 28 May 2021).

17. Available online: https:// oem.bmj.com $\backslash \mathrm{T} 1 \backslash$ guilsinglrightinline-supplementary-material-2 (accessed on 20 August 2021).

18. Deng, K.; Yang, C.; Tan, Q.; Song, W.; Lu, M.; Zhao, W.; Lou, G.; Li, Z.; Li, K.; Hou, Y. Sites of distant metastases and overall survival in ovarian cancer: A study of 1481 patients. Gynecol. Oncol. 2018, 150, 460-465. [CrossRef]

19. Sehouli, J.; Olschewski, J.; Schotters, V.; Fotopoulou, C.; Pietzner, K. Prognostic role of early versus late onset of bone metastasis in patients with carcinoma of the ovary, peritoneum and fallopian tube. Ann. Oncol. 2013, 24, 3024-3028. [CrossRef] [PubMed]

20. Zhang, M.; Sun, J. Bone metastasis from ovarian cancer. Clinical analysis of 26 cases. Saudi Med. J. 2013, 34, 1270-1273. [PubMed] 
21. Zhang, C.; Guo, X.; Peltzer, K.; Ma, W.; Qi, L.; Zhang, Y.; Han, X.; Baklaushev, V.P.; Yao, Y.; Wang, G.; et al. The prevalence, associated factors for bone metastases development and prognosis in newly diagnosed ovarian cancer: A large population based real-world study. J. Cancer 2019, 10, 3133-3139. [CrossRef]

22. Guo, J.; Cui, X.; Zhang, X.; Qian, H.; Duan, H.; Zhang, Y. The Clinical Characteristics of Endometrial Cancer With Extraperitoneal Metastasis and the Value of Surgery in Treatment. Technol. Cancer Res. Treat 2020, 19, 1533033820945784. [CrossRef]

23. Hong, L.; Cristiano, L.; Peters, E.; Ioffe, Y. Detection of bone metastases in uterine cancer: How common are they and should PET/CT be the standard for diagnosis? Gynecol. Oncol. Rep. 2021, 35, 100698. [CrossRef]

24. Kimyon, G.; Karalok, A.; Basaran, D.; Ureyen, I.; Celik, M.; Tasci, T.; Tulunay, G.; Turan, T. Bone recurrence rarely seen in endometrial cancer and review of the literature. J. Obstet. Gynaecol. Res. 2016, 42, 602-611. [CrossRef]

25. Li, H.; Zhang, R.; Chen, C.; Wu, C.; Lin, H.; Li, J.; Lin, Z. Prognostic value of different metastatic sites for patients with FIGO stage IVB endometrial cancer after surgery: A SEER database analysis. J. Surg. Oncol. 2020, 122, 941-948. [CrossRef] [PubMed]

26. Liu, Y.; Chi, S.; Zhou, X.; Zhao, R.; Xiao, C.; Wang, H. Prognostic value of distant metastatic sites in stage IV endometrial cancer: A SEER database study of 2948 women. Int. J. Gynaecol. Obstet. 2020, 149, 16-23. [CrossRef]

27. Mao, W.; Wei, S.; Yang, H.; Yu, Q.; Xu, M.; Guo, J.; Gao, L. Clinicopathological study of organ metastasis in endometrial cancer. Future Oncol. 2020, 16, 525-540. [CrossRef] [PubMed]

28. McEachron, J.; Chatterton, C.; Hastings, V.; Gorelick, C.; Economos, K.; Lee, Y.C.; Kanis, M.J. A clinicopathologic study of endometrial cancer metastatic to bone: Identification of microsatellite instability improves treatment strategies. Gynecol. Oncol. Rep. 2020, 32, 100549. [CrossRef]

29. Ouldamer, L.; Bendifallah, S.; Body, G.; Touboul, C.; Graesslin, O.; Raimond, E.; Collinet, P.; Coutant, C.; Bricou, A.; Lavoué, V.; et al. Incidence, patterns and prognosis of first distant recurrence after surgically treated early stage endometrial cancer: Results from the multicentre FRANCOGYN study group. Eur. J. Surg. Oncol. 2019, 45, 672-678. [CrossRef]

30. Takeshita, S.; Todo, Y.; Matsumiya, H.; Okamoto, K.; Yamashiro, K.; Kato, H. A prediction model of survival for patients with bone metastasis from uterine corpus cancer. Jpn. J. Clin. Oncol. 2016, 46, 973-978. [CrossRef]

31. Uccella, S.; Morris, J.M.; Bakkum-Gamez, J.N.; Keeney, G.L.; Podratz, K.C.; Mariani, A. Bone metastases in endometrial cancer: Report on 19 patients and review of the medical literature. Gynecol. Oncol. 2013, 130, 474-482. [CrossRef]

32. Yoon, A.; Choi, C.H.; Kim, T.H.; Choi, J.K.; Park, J.Y.; Lee, Y.Y.; Kim, T.J.; Lee, J.W.; Bae, D.S.; Kim, B.G. Bone metastasis in primary endometrial carcinoma: Features, outcomes, and predictors. Int. J. Gynecol. Cancer 2014, 24, 107-112. [CrossRef] [PubMed]

33. Bartosch, C.; Afonso, M.; Pires-Luís, A.S.; Galaghar, A.; Guimarães, M.; Antunes, L.; Lopes, J.M. Distant Metastases in Uterine Leiomyosarcomas: The Wide Variety of Body Sites and Time Intervals to Metastatic Relapse. Int. J. Gynecol. Pathol. 2017, 36, 31-41. [CrossRef]

34. Tirumani, S.H.; Deaver, P.; Shinagare, A.B.; Tirumani, H.; Hornick, J.L.; George, S.; Ramaiya, N.H. Metastatic pattern of uterine leiomyosarcoma: Retrospective analysis of the predictors and outcome in 113 patients. J. Gynecol. Oncol. 2014, $25,306-312$. [CrossRef] [PubMed]

35. Prieske, K.; Haeringer, N.; Grimm, D.; Trillsch, F.; Eulenburg, C.; Burandt, E.; Schmalfeldt, B.; Mahner, S.; Mueller, V.; Woelber, L. Patterns of distant metastases in vulvar cancer. Gynecol. Oncol. 2016, 142, 427-434. [CrossRef] [PubMed]

36. Kanayama, T.; Mabuchi, S.; Shimura, K.; Hisamatsu, T.; Isohashi, F.; Hamasaki, T.; Kimura, T. Prognostic factors for survival in cervical cancer patients with bone metastasis. Eur. J. Gynaecol. Oncol. 2015, 36, 290-293. [PubMed]

37. Kocaer, M.; Gülseren, V.; Özdemir, I.A.; Güngördük, O.; Mart, E.M.; Sanci, M.; Güngördük, K. Management of Vertebral Metastasis in Patients With Uterine Cervical Cancer. Int. J. Gynecol. Cancer 2018, 28, 1191-1195. [CrossRef] [PubMed]

38. Lin, A.; Ma, S.; Dehdashti, F.; Markovina, S.; Schwarz, J.; Siegel, B.; Powell, M.; Grigsby, P. Detection of distant metastatic disease by positron emission tomography with 18 F-fluorodeoxyglucose (FDG-PET) at initial staging of cervical carcinoma. Int. J. Gynecol. Cancer 2019, 29, 487-491. [CrossRef]

39. Makino, H.; Nishio, S.; Tsubamoto, H.; Shimada, M.; Nishikawa, R.; Kai, K.; Ito, K.; Mizuno, T.; Ushijima, K.; Morishige, K.I. Treatment and prognosis of bone metastasis from cervical cancer (KCOG-G1202s). J. Obstet. Gynaecol. Res. 2016, 42, 701-706. [CrossRef]

40. Manders, D.B.; Sims, T.T.; Albuquerque, K.V.; Carlson, M.J.; Richardson, D.L.; Kehoe, S.M.; Miller, D.S.; Lea, J.S. Emphasis on Systemic Therapy in Women with Pelvic Bone Metastasis at Time of Diagnosis of Cervical Cancer. Am. J. Clin. Oncol. 2018, 41, 1137-1141. [CrossRef]

41. Matsumiya, H.; Todo, Y.; Okamoto, K.; Takeshita, S.; Yamazaki, H.; Yamashiro, K.; Kato, H. A prediction model of survival for patients with bone metastasis from uterine cervical cancer. J. Gynecol. Oncol. 2016, 27, e55. [CrossRef]

42. Nartthanarung, A.; Thanapprapasr, K.; Udomsubpayakul, U.; Thanapprapasr, D. Age and survival of cervical cancer patients with bone metastasis. Asian Pac. J. Cancer Prev. 2014, 15, 8401-8404. [CrossRef]

43. Sethi, S.; Ramalingam, V.; Yadav, M.; Thulkar, S. Nodal metastasis of cervix carcinoma- As a probable cause of bone erosion. J. Cancer Res. Ther. 2019, 15, 1260-1264.

44. Yin, Z.; Lou, H.; Tang, H.; Ni, J.; Zhou, Q.; Chen, M. Efficacy of radical doses of pelvic radiotherapy for primary tumor treatment in patients with newly diagnosed organ metastatic cervical cancer. Radiat. Oncol. 2019, 14, 82. [CrossRef] [PubMed]

45. Yoon, A.; Choi, C.H.; Kim, H.J.; Park, J.Y.; Lee, Y.Y.; Kim, T.J.; Lee, J.W.; Bae, D.S.; Kim, B.G. Contributing factors for bone metastasis in uterine cervical cancer. Int. J. Gynecol. Cancer 2013, 23, 1311-1317. [CrossRef] 
46. Zhang, Y.; Guo, X.; Wang, G.; Ma, W.; Liu, R.; Han, X.; Li, L.; Baklaushev, V.P.; Bryukhovetskiy, A.S.; Wang, W.; et al. Real-World Study of the Incidence, Risk Factors, and Prognostic Factors Associated with Bone Metastases in Women with Uterine Cervical Cancer Using Surveillance, Epidemiology, and End Results (SEER) Data Analysis. Med. Sci. Monit. 2018, 24, 6387-6397. [CrossRef] [PubMed]

47. Zhang, Y.; Guo, Y.; Zhou, X.; Wang, X.; Wang, X. Prognosis for different patterns of distant metastases in patients with uterine cervical cancer: A population-based analysis. J. Cancer 2020,11, 1532-1541. [CrossRef]

48. Zhou, S.; Peng, F. Patterns of metastases in cervical cancer: A population-based study. Int. J. Clin. Exp. Pathol. 2020, 13, 1615-1623. [PubMed]

49. Liu, A.Y.; Roudier, M.P.; True, L.D. Heterogeneity in primary and metastatic prostate cancer as defined by cell surface CD profile. Am. J. Pathol. 2004, 165, 1543-1556. [CrossRef]

50. Roudier, M.P.; Corey, E.; True, L.D.; Hiagno, C.S.; Ott, S.M.; Vessell, R.L. Histological, immunophenotypic and histomorphometric characterization of prostate cancer bone metastases. Cancer Treat Res. 2004, 118, 311-339. [PubMed]

51. Coleman, R.E.; Croucher, P.I.; Padhani, A.R.; Clézardin, P.; Chow, E.; Fallon, M.; Guise, T.; Colangeli, S.; Capanna, R.; Costa, L. Bone metastases. Nat. Rev. Dis. Primers 2020, 6, 83. [CrossRef] [PubMed]

52. Yin, J.J.; Pollock, C.B.; Kelly, K. Mechanisms of cancer metastasis to the bone. Cell Res. 2005, 15, 57-62. [CrossRef]

53. Desautels, D.N.; Harlos, C.H.; Jerzak, K.J. Role of bone-modifying agents in advanced cancer. Ann. Palliat. Med. 2020, 9, 1314-1323. [CrossRef]

54. Mullen, M.M.; Divine, L.M.; Porcelli, B.P.; Wilkinson-Ryan, I.; Dans, M.C.; Powell, M.A.; Mutch, D.G.; Hagemann, A.R.; Thaker, P.H. The effect of a multidisciplinary palliative care initiative on the end of life care in gynecologic oncology patients. Gynecol. Oncol. 2017, 147, 460-464. [CrossRef]

55. Lopez-Acevedo, M.; Lowery, W.J.; Lowery, A.W.; Lee, P.S.; Havrilesky, L.J. Palliative and hospice care in gynecologic cancer: A review. Gynecol. Oncol. 2013, 131, 215-221. [CrossRef]

56. Zhang, R.; Shi, H.; Ren, F.; Feng, W.; Cao, Y.; Li, G.; Liu, Z.; Ji, P.; Zhang, M. MicroRNA-338-3p suppresses ovarian cancer cells growth and metastasis: Implication of Wnt/catenin beta and MEK/ERK signaling pathways. J. Exp. Clin. Cancer Res. 2019, 38, 494. [CrossRef]

57. Mei, J.; Huang, Y.; Hao, L.; Liu, Y.; Yan, T.; Qiu, T.; Xu, R.; Xu, B.; Xiao, Z.; Jiang, X.; et al. DAAM1-mediated migration and invasion of ovarian cancer cells are suppressed by miR-208a-5p. Pathol. Res. Pract. 2019, 215, 152452. [CrossRef] [PubMed]

58. Ebrahimi, S.O.; Reiisi, S. Downregulation of miR-4443 and miR-5195-3p in ovarian cancer tissue contributes to metastasis and tumorigenesis. Arch. Gynecol. Obstet. 2019, 299, 1453-1458. [CrossRef]

59. Cohen, P.A.; Flowers, N.; Tong, S.; Hannan, N.; Pertile, M.D.; Hui, L. Abnormal plasma DNA profiles in early ovarian cancer using a non-invasive prenatal testing platform: Implications for cancer screening. BMC Med. 2016, 14, 126. [CrossRef] [PubMed]

60. Vanderstichele, A.; Busschaert, P.; Smeets, D.; Landolfo, C.; Nieuwenhuysen, E.V.; Leunen, K.; Neven, P.; Amant, F.; Mahner, S.; Braicu, E.I.; et al. Chromosomal Instability in Cell-Free DNA as a Highly Specific Biomarker for Detection of Ovarian Cancer in Women with Adnexal Masses. Clin. Cancer Res. 2017, 23, 2223-2231. [CrossRef] [PubMed]

61. Harris, F.R.; Kovtun, I.V.; Smadbeck, J.; Multinu, F.; Jatoi, A.; Kosari, F.; Kalli, K.R.; Murphy, S.J.; Halling, G.C.; Johnson, S.H.; et al. Quantification of Somatic Chromosomal Rearrangements in Circulating Cell-Free DNA from Ovarian Cancers. Sci. Rep. 2016, 6, 29831. [CrossRef] [PubMed]

62. Tugwell, P.; Tovey, D. PRISMA 2020. J. Clin. Epidemiol. 2021, 134, A5-A6. [CrossRef] [PubMed] 